\title{
Bis(imino)pyridine Iron(II) Alkyl Cations for Olefin Polymerization.
}

\author{
Marco W. Bouwkamp, Emil Lobkovsky and Paul J. Chirik* \\ Department of Chemistry and Chemical Biology, Baker Laboratory, \\ Cornell University, Ithaca, New York 14853 \\ - Supporting Information -
}




\section{Table of Contents}

$\begin{array}{ll}\text { Experimental Procedures } & \text { S3 }\end{array}$

Crystallographic Data for $\left[\left({ }^{\mathrm{iPr}} \mathrm{PDI}\right) \mathrm{Fe}\left(\mathrm{CH}_{2} \mathrm{SiMe}_{3}\right)\left(\mathrm{Et}_{2} \mathrm{O}\right)\right]\left[\mathrm{BPh}_{4}\right]\left(\mathbf{2}-\mathbf{E t}_{\mathbf{2}} \mathbf{O}\right) \quad \mathrm{S} 8$

Crystallographic Data for $\left[\left({ }^{i \mathrm{Pr}} \mathrm{PDI}\right) \mathrm{Fe}\left(\mathrm{CH}_{2} \mathrm{SiMe}_{3}\right)(\mathrm{THF})\right]\left[\mathrm{BPh}_{4}\right](\mathbf{2}$-THF) $\quad \mathrm{S} 13$

Crystallographic Data for $\left[\left({ }^{i \mathrm{Pr}} \mathrm{PDI}\right) \mathrm{Fe}\left(\mathrm{CH}_{2} \mathrm{SiMe}_{2} \mathrm{CH}_{2} \mathrm{SiMe}_{3}\right)\right]\left[\mathrm{MeB}\left(\mathrm{C}_{6} \mathrm{~F}_{5}\right)_{3}\right](\mathbf{3}) \quad \mathrm{S} 18$ 


\section{Experimental Procedures}

General Considerations. All air- and moisture-sensitive manipulations were carried out using standard vacuum line, Schlenk and cannula techniques or in an MBraun inert atmosphere drybox containing an atmosphere of purified nitrogen. The MBraun drybox was equipped with a cold well designed for freezing samples in liquid nitrogen. Solvents for air- and moisture-sensitive manipulations were initially dried and deoxygenated using literature procedures. ${ }^{1}$ Argon and hydrogen gas were purchased from Airgas Incorporated and passed through a column containing manganese oxide supported on vermiculite and 4 $\AA$ molecular sieves before admission to the high vacuum line. Benzene- $d_{6}$ was purchased from Cambridge Isotope Laboratories and distilled from sodium metal under an atmosphere of argon and stored over $4 \AA$ molecular sieves or sodium metal. Bromobenzene- $d_{5}$ was purchased from Cambridge Isotope Laboratories and dried over 4 $\AA$ A molecular sieves. Ethylene and methylalumoxane were purchased from Akzo Nobel. The toluene was removed from the MAO and the resulting white powder was dried under high vacuum for 24 hours. The ethylene was dried by passage through a column packed with activated molecular sieves ( $4 \AA$ ) and freshly activated manganese oxide on vermiculite. $\mathrm{B}\left(\mathrm{C}_{6} \mathrm{~F}_{5}\right)_{3}$ was purchased from Strem; $\left[\mathrm{PhNMe}_{2} \mathrm{H}\right]\left[\mathrm{BPh}_{4}\right]^{2}$ and 1$\left(\mathrm{CH}_{2} \mathrm{SiMe}_{3}\right)_{2}{ }^{3}$ were prepared according to literature procedures.

${ }^{1} \mathrm{H}$ NMR spectra were recorded on Varian Inova 400 and 500 spectrometers operating at 299.763, 399.780 and $500.62 \mathrm{MHz}$, respectively. All chemical shifts are reported relative to $\mathrm{SiMe}_{4}$ using ${ }^{1} \mathrm{H}$ (residual) chemical shifts of the solvent as a secondary standard. For paramagnetic molecules, the ${ }^{1} \mathrm{H}$ NMR data are reported with the 
chemical shift followed by the peak width at half height in Hertz or multiplicity, followed by integration value and where possible, peak assignment. ${ }^{19} \mathrm{~F}$ NMR spectra were recorded on Varian Inova 400 spectrometer operating at $470.986 \mathrm{MHz}$ and ${ }^{13} \mathrm{C}$ NMR spectra were recorded on Varian Inova 500 spectrometer operating at $125.892 \mathrm{MHz}$.

Single crystals suitable for X-ray diffraction were coated with polyisobutylene oil in a drybox and were quickly transferred to the goniometer head of a Siemens SMART CCD Area detector system equipped with a molybdenum X-ray tube $(\lambda=0.71073 \AA)$. Preliminary data revealed the crystal system. A hemisphere routine was used for data collection and determination of lattice constants. The space group was identified and the data were processed using the Bruker SAINT program and corrected for absorption using SADABS. The structures were solved using direct methods (SHELXS) completed by subsequent Fourier synthesis and refined by full-matrix least-squares procedures.

Polymer melting temperatures were measured with a TA Instruments DSC Q100. The sample run was performed from -30 to $200{ }^{\circ} \mathrm{C}$ with a heating rate of $10{ }^{\circ} \mathrm{C}$ per minute. The linearity and end groups of the polymers were determined by ${ }^{1} \mathrm{H}$ and ${ }^{13} \mathrm{C}$ NMR spectroscopy in $\mathrm{CD}_{2} \mathrm{Cl}_{4}$ at $120{ }^{\circ} \mathrm{C}$. Molecular weights $(M \mathrm{n}$ and $M \mathrm{w})$ and polydispersities $(M \mathrm{w} / M \mathrm{n})$ were determined by high temperature gel-permeation chromatography (GPC). Analyses were performed with a Waters Alliance GPCV 2000 gel permeation chromatograph equipped with a Waters DRI detector and viscometer. The column set (four Waters HT 6E and one Waters HT 2) was eluted with 1,2,4trichlorobenzene containing $0.01 \mathrm{wt} \%$ di-tert-butylhydroxytoluene (BHT) at $1.0 \mathrm{~mL} / \mathrm{min}$ at $140{ }^{\circ} \mathrm{C}$. Data were calibrated using four monomodal polyethylene standards (from Polymer Standards Service) which generated a calibration curve with twelve data points. 
Polymer solutions were usually placed in a $140{ }^{\circ} \mathrm{C}$ oven for $24 \mathrm{~h}$ prior to molecular weight measurements.

Preparation of $\left.\left[{ }^{\mathrm{iPr}} \mathrm{PDI}\right) \mathrm{Fe}\left(\mathrm{CH}_{2} \mathrm{SiMe}_{3}\right)\right]\left[\mathrm{BPh}_{4}\right](2)$. A solution of $0.059 \mathrm{~g}(0.083 \mathrm{mmol})$ of $\mathbf{1}-\left(\mathbf{C H}_{2} \mathbf{S i M e}_{3}\right)_{2}$ in approximately $5 \mathrm{~mL}$ of toluene was added to $0.030 \mathrm{~g}(0.073 \mathrm{mmol})$ of $\left[\mathrm{PhMe}_{2} \mathrm{NH}\right]\left[\mathrm{BPh}_{4}\right]$. The resulting mixture was stirred for 3 hours, affording a dark brown oil. Triturating with approximately $10 \mathrm{~mL}$ of pentane $(10 \mathrm{~mL})$ furnishes a solid compound. The resulting solid was washed by pentane and dried in vacuo to furnish $0.034 \mathrm{~g}(49 \%)$ of $2 .{ }^{1} \mathrm{H}$ NMR (benzene- $\left.d_{6}, 400 \mathrm{MHz}, \mathrm{RT}\right) \delta=-101.18$ (570.68), - 39.99 (219.51), -19.21 (49.17), -15.81 (130.33), -2.61 (3483.99), 7.35 (partially overlapping with $\left.\mathrm{C}_{6} \mathrm{D}_{6}\right), 10.64$ (207.16), 17.00 (272.97), 49.90 (167.67), 76.53 (177.43), 127.85 (243.45). Due to the poor solubility of the compound in $\mathrm{C}_{6} \mathrm{D}_{6}$, the ${ }^{1} \mathrm{H}$ NMR spectrum does not allow accurate integration of the signals, prohibiting definitive assignment.

Preparation of $\left[\left({ }^{\mathrm{iPr}} \mathrm{PDI}\right) \mathrm{Fe}\left(\mathrm{CH}_{2} \mathrm{SiMe}_{3}\right)(\mathrm{THF})\right]\left[\mathrm{BPh}_{4}\right](\mathbf{2}-\mathrm{THF})$. A solution of $0.051 \mathrm{~g}$ $(0.072 \mathrm{mmol})$ of $\mathbf{1}-\left(\mathbf{C H}_{2} \mathbf{S i M e}_{3}\right)_{2}$ in $5 \mathrm{~mL}$ of THF was added to $0.034 \mathrm{mg}(77 \mathrm{mmol})$ of $\left[\mathrm{PhNMe}_{2} \mathrm{H}\right]\left[\mathrm{BPh}_{4}\right]$. After 10 minutes, the solution was layered with pentane $(10 \mathrm{~mL})$ furnishing $0.054 \mathrm{~g}(74 \%)$ of red crystals identified as 2-THF. Analysis for $\mathrm{C}_{65} \mathrm{H}_{82} \mathrm{BFeN}_{3} \mathrm{OSi}$. Calcd: C, 76.83; H, 8.13; N, 4.14. Found: C, 76.48; H, 7.98; N, 3.82Magnetic moment (Gouy): $\mu_{\text {eff }}=4.6 \mu_{\mathrm{B}} .{ }^{1} \mathrm{H}$ NMR (benzene- $d_{6,}, 400 \mathrm{MHz}, \mathrm{RT}$ ) $\delta=-$ 73.68 (315.18), -26.47 (937.41), -13.09 (771.28), -8.99 (190.15), -2.76 (375.82), 7.86 (56.50, $\left.\mathrm{BPh}_{4}\right), 8.76\left(44.58, \mathrm{BPh}_{4}\right), 10.26\left(33.67, \mathrm{BPh}_{4}\right), 19.80$ (1289.62), 38.37 (820.94), 52.66 (953.58), 88.13 (291.21), 113.83 (484.37). The ${ }^{1} \mathrm{H}$ NMR spectrum exhibits 
overlapping signals prohibiting integration of the signals and with that definitive assignment.

$\left[\left({ }^{i \mathrm{Pr}} \mathrm{PDI}\right) \mathrm{Fe}\left(\mathrm{CH}_{2} \mathrm{SiMe}_{3}\right)\left(\mathbf{E t}_{2} \mathbf{O}\right)\right]\left[\mathrm{BPh}_{4}\right]$. This molecule was prepared in a similar manner to $\mathbf{2}$-THF with $0.082 \mathrm{~g}(0.12 \mathrm{mmol})$ of $\mathbf{1 -}\left(\mathbf{C H}_{2} \mathbf{S i M e}_{3}\right)_{2}$ and $0.046 \mathrm{mg}(0.11 \mathrm{mmol})$ of $\left[\mathrm{PhNMe}_{2} \mathrm{H}\right]\left[\mathrm{BPh}_{4}\right]$ yielding $0.062 \mathrm{~g}(54 \%)$ of red-brown crystals identified as $\mathbf{2}-\mathbf{E} \mathbf{t}_{\mathbf{2}} \mathbf{O}$. Analysis for $\mathrm{C}_{65} \mathrm{H}_{84} \mathrm{BFeN}_{3} \mathrm{OSi}$. Calcd: C, 76.68; H, 8.32; N, 4.13. Found: C, 76.38; H, 7.92; N, 4.41. Magnetic moment (Gouy): $\mu_{\text {eff }}=4.8 \mu_{\mathrm{B}} \cdot{ }^{1} \mathrm{H}$ NMR (benzene- $d_{6}, 400 \mathrm{MHz}$, RT) $\delta=-99.0(692.8,6 \mathrm{H}, \mathrm{N}=\mathrm{CMe}),-40.3\left(209.4,12 \mathrm{H}, \mathrm{CH} \mathrm{Me}_{2}\right),-19.0(46.5,2 \mathrm{H}),-14.9$ (83.3, 12H, $\mathrm{CHMe} 2), 6.6\left(88.6,4 \mathrm{H}, \mathrm{BPh}_{4}\right), 13.2\left(105.3,8 \mathrm{H}, \mathrm{BPh}_{4}\right), 21.5\left(86.8,8 \mathrm{H}, \mathrm{BPh}_{4}\right)$, $50.2(212.4,4 \mathrm{H}), 78.8(153.2,4 \mathrm{H}), 130.4(214.8,1 \mathrm{H}), 3$ resonances not located.

Preparation of $\left[\left({ }^{i \mathrm{Pr}} \mathrm{PDI}\right) \mathrm{Fe}\left(\mathrm{CH}_{2} \mathrm{SiMe}_{2} \mathrm{CH}_{2} \mathrm{SiMe}_{3}\right)\right]\left[\mathrm{MeB}\left(\mathrm{C}_{6} \mathrm{~F}_{5}\right)_{3}\right]$ (3). A solution containing $0.052 \mathrm{~g}(0.10 \mathrm{mmol})$ of $\mathrm{B}\left(\mathrm{C}_{6} \mathrm{~F}_{5}\right)_{3}$ in approximately $10 \mathrm{~mL}$ of pentane was added to $0.075 \mathrm{mg}$ of $\mathbf{1}$ also dissolved in approximately $10 \mathrm{~mL}$ of pentane. The reaction mixture was allowed to stand, without stirring, for one hour after which time a red crystalline precipitate formed. The crystals were collected by filtration and washing with pentane to yield $0.048 \mathrm{~g}(39 \%)$ of a red solid identified as 3. Analysis for $\mathrm{C}_{59} \mathrm{H}_{65} \mathrm{BF}_{15} \mathrm{FeN}_{3} \mathrm{Si}_{2}$. Calcd: C, 57.90; H, 5.35; N, 3.43. Found: C, 57.90; H, 5.00; N, 3.04. Magnetic moment (Gouy): $\mu_{\mathrm{eff}}=4.8 \mu_{\mathrm{B}} \cdot{ }^{1} \mathrm{H}$ NMR (benzene- $d_{6}, 400 \mathrm{MHz}, \mathrm{RT}$ ) $\delta=-97.4$ $(584.0,6 \mathrm{H}, \mathrm{N}=\mathrm{CMe}),-42.0\left(280.4,12 \mathrm{H}, \mathrm{CH} \mathrm{Me}_{2}\right),-20.2(31.6,2 \mathrm{H}),-16.5(70.5,12 \mathrm{H}$, $\left.\mathrm{CHMe} e_{2}\right), \quad-0.8 \quad\left(35.3, \quad 3 \mathrm{H}, \quad \mathrm{FeCH}_{2} \mathrm{SiMe}_{2} \mathrm{CH}_{2} \mathrm{SiMe}_{3}\right), \quad 13.9 \quad(25.7, \quad 9 \mathrm{H}$, $\left.\mathrm{FeCH}_{2} \mathrm{SiMe}_{2} \mathrm{CH}_{2} \mathrm{SiMe} e_{3}\right), 20.4$ (43.8, 3H, $\left.\mathrm{FeCH}_{2} \mathrm{SiMe}_{2} \mathrm{CH}_{2} \mathrm{SiMe}_{3}\right), 53.7$ (221.8, 4H), 71.0 
$(100.0,4 \mathrm{H}), 126.15(186.5,1 \mathrm{H}, \mathrm{p}-\mathrm{py}), 3$ resonances not located. ${ }^{19} \mathrm{~F}$ NMR (toluene- $d_{8}$, $400 \mathrm{MHz}, \mathrm{RT}) \delta=-172.5(3 \mathrm{~F}, p-\mathrm{F}),-166.5(6 \mathrm{~F}, m-\mathrm{F}),-133.41(6 \mathrm{~F}, o-\mathrm{F})$.

General Ethylene Polymerization Procedure. The desired iron complex (and MAO if necessary) were charged into a scintillation vial and washed into a $250 \mathrm{~mL}$ round bottomed flask with $10 \mathrm{~mL}$ of toluene. The flask was equipped with a $180^{\circ}$ needle valve and attached to a high vacuum line, frozen in liquid nitrogen and degassed. The reaction mixture was thawed and stirred for 10 minutes to equilibrate. The manifold was charged with 1 atm of dry ethylene and the needle valve opened. The polymerization was stirred for the desired time, typically five minutes, and then quenched with $\mathrm{MeOH} / \mathrm{HCl}$. The polymer was collected by filtration and dried under reduced pressure. 
a)

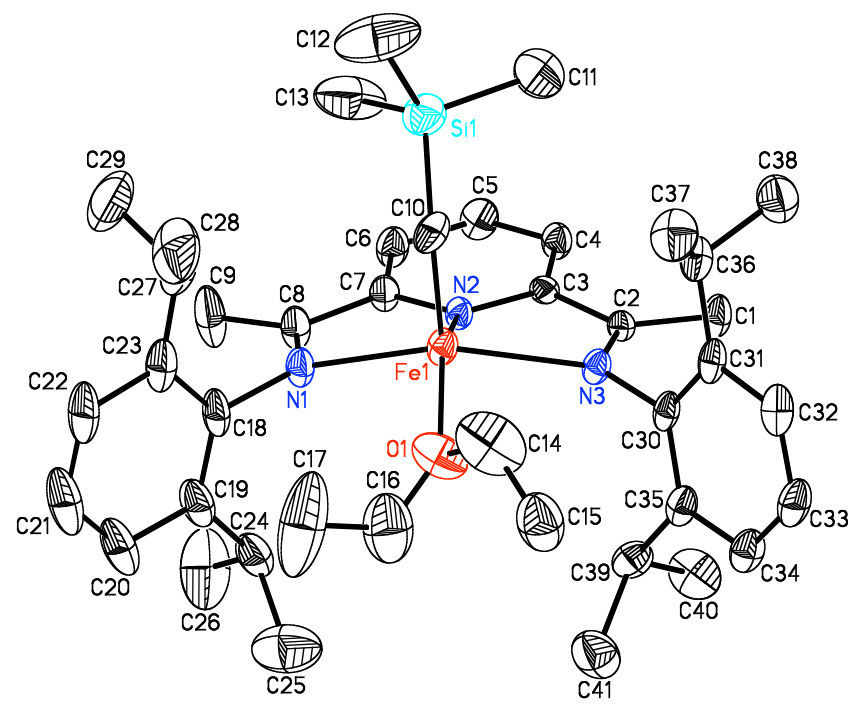

b)

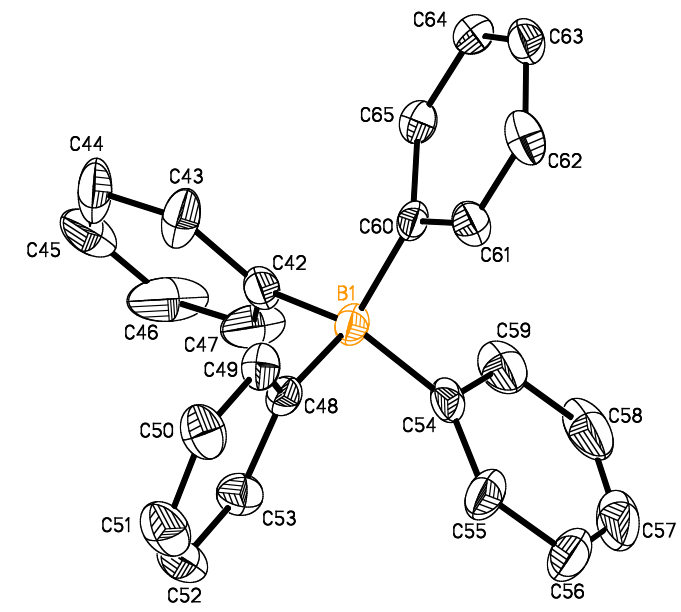

Figure S1. Fully labeled view of a) the cation and b) the anion of $\mathbf{2}-\mathbf{E t}_{\mathbf{2}} \mathbf{O}$ at $30 \%$ probability ellipsoids. Hydrogen atoms omitted for clarity. 
Table S1. Crystal data and structure refinement for $\mathbf{2}-\mathbf{E t}_{\mathbf{2}} \mathbf{O}$.

Identification code

Empirical formula

Formula weight

Temperature

Wavelength

Crystal system

Space group

Unit cell dimensions

Volume

Z

Density (calculated)

Absorption coefficient

$\mathrm{F}(000)$

Crystal size

Theta range for data collection

Index ranges

Reflections collected

Independent reflections

Completeness to theta $=23.31^{\circ}$

Absorption correction

Max. and min. transmission

Refinement method

Data / restraints / parameters

Goodness-of-fit on $\mathrm{F}^{2}$

Final $\mathrm{R}$ indices [I $>2 \operatorname{sigma}(\mathrm{I})]$

$\mathrm{R}$ indices (all data)

Largest diff. peak and hole mwb13

C69 H82 B Fe N3 O2 Si

1080.13

173(2) K

$0.71073 \AA$

Monoclinic

$\mathrm{P} 2(1) / \mathrm{n}$

$\mathrm{a}=12.9630(6) \AA \quad \alpha=90^{\circ}$.

$\mathrm{b}=22.7555(10) \AA \quad \beta=99.957(1)^{\circ}$.

$\mathrm{c}=22.4921(11) \AA \quad \gamma=90^{\circ}$.

$6534.8(5) \AA^{3}$

4

$1.098 \mathrm{Mg} / \mathrm{m}^{3}$

$0.292 \mathrm{~mm}^{-1}$

2312

$0.50 \times 0.10 \times 0.05 \mathrm{~mm}^{3}$

1.70 to $23.31^{\circ}$.

$-14<=\mathrm{h}<=14,-25<=\mathrm{k}<=21,-24<=1<=24$

44082

$9418[\mathrm{R}($ int $)=0.0606]$

$99.6 \%$

Semi-empirical from equivalents

0.9855 and 0.8676

Full-matrix least-squares on $\mathrm{F}^{2}$

9418 / 0 / 694

1.016

$\mathrm{R} 1=0.0725, \mathrm{wR} 2=0.1879$

$\mathrm{R} 1=0.1191, \mathrm{wR} 2=0.2142$

0.859 and -0.513 e. $\AA^{-3}$ 
Table S2. Bond lengths $[\AA]$ and angles $\left[{ }^{\circ}\right]$ for $\mathbf{2}-\mathbf{E t}_{2} \mathbf{O}$.

\begin{tabular}{|c|c|c|c|}
\hline $\mathrm{Fe}(1)-\mathrm{C}(10)$ & $2.088(5)$ & $\mathrm{C}(22)-\mathrm{C}(23)$ & $1.407(8)$ \\
\hline $\mathrm{Fe}(1)-\mathrm{N}(2)$ & $2.087(3)$ & $C(23)-C(27)$ & $1.485(9)$ \\
\hline $\mathrm{Fe}(1)-\mathrm{O}(1)$ & $2.122(4)$ & $C(24)-C(26)$ & $1.509(8)$ \\
\hline $\mathrm{Fe}(1)-\mathrm{N}(3)$ & $2.297(3)$ & $C(24)-C(25)$ & $1.515(9)$ \\
\hline $\mathrm{Fe}(1)-\mathrm{N}(1)$ & $2.319(4)$ & $C(27)-C(28)$ & $1.532(9)$ \\
\hline $\operatorname{Si}(1)-C(10)$ & $1.808(6)$ & $C(27)-C(29)$ & $1.545(9)$ \\
\hline $\operatorname{Si}(1)-C(11)$ & $1.813(8)$ & $\mathrm{C}(30)-\mathrm{C}(31)$ & $1.393(7)$ \\
\hline $\mathrm{Si}(1)-\mathrm{C}(12)$ & $1.839(7)$ & $\mathrm{C}(30)-\mathrm{C}(35)$ & $1.401(7)$ \\
\hline $\operatorname{Si}(1)-C(13)$ & $1.864(7)$ & $\mathrm{C}(31)-\mathrm{C}(32)$ & $1.402(7)$ \\
\hline $\mathrm{O}(1)-\mathrm{C}(16)$ & $1.284(9)$ & $\mathrm{C}(31)-\mathrm{C}(36)$ & $1.509(7)$ \\
\hline $\mathrm{O}(1)-\mathrm{C}(14)$ & $1.516(9)$ & $\mathrm{C}(32)-\mathrm{C}(33)$ & $1.374(8)$ \\
\hline $\mathrm{N}(1)-\mathrm{C}(8)$ & $1.282(6)$ & $\mathrm{C}(33)-\mathrm{C}(34)$ & $1.378(7)$ \\
\hline N(1)-C(18) & $1.470(6)$ & $\mathrm{C}(34)-\mathrm{C}(35)$ & $1.397(6)$ \\
\hline$N(2)-C(7)$ & $1.340(5)$ & $\mathrm{C}(35)-\mathrm{C}(39)$ & $1.516(7)$ \\
\hline $\mathrm{N}(2)-\mathrm{C}(3)$ & $1.349(5)$ & $\mathrm{C}(36)-\mathrm{C}(38)$ & $1.528(8)$ \\
\hline $\mathrm{N}(3)-\mathrm{C}(2)$ & $1.290(5)$ & $\mathrm{C}(36)-\mathrm{C}(37)$ & $1.537(7)$ \\
\hline $\mathrm{N}(3)-\mathrm{C}(30)$ & $1.443(5)$ & $\mathrm{C}(39)-\mathrm{C}(40)$ & $1.503(8)$ \\
\hline $\mathrm{C}(1)-\mathrm{C}(2)$ & $1.487(6)$ & $\mathrm{C}(39)-\mathrm{C}(41)$ & $1.534(8)$ \\
\hline$C(2)-C(3)$ & $1.484(6)$ & $\mathrm{B}(1)-\mathrm{C}(42)$ & $1.629(7)$ \\
\hline $\mathrm{C}(3)-\mathrm{C}(4)$ & $1.372(6)$ & $\mathrm{B}(1)-\mathrm{C}(60)$ & $1.633(7)$ \\
\hline$C(4)-C(5)$ & $1.384(6)$ & $\mathrm{B}(1)-\mathrm{C}(54)$ & $1.652(7)$ \\
\hline$C(5)-C(6)$ & $1.375(6)$ & $\mathrm{B}(1)-\mathrm{C}(48)$ & $1.646(7)$ \\
\hline$C(6)-C(7)$ & $1.376(6)$ & $\mathrm{C}(42)-\mathrm{C}(43)$ & $1.383(7)$ \\
\hline$C(7)-C(8)$ & $1.487(6)$ & $\mathrm{C}(42)-\mathrm{C}(47)$ & $1.388(7)$ \\
\hline$C(8)-C(9)$ & $1.501(7)$ & $\mathrm{C}(43)-\mathrm{C}(44)$ & $1.385(8)$ \\
\hline$C(14)-C(15)$ & $1.424(11)$ & $\mathrm{C}(44)-\mathrm{C}(45)$ & $1.357(10)$ \\
\hline$C(16)-C(17)$ & $1.597(11)$ & $C(45)-C(46)$ & $1.373(12)$ \\
\hline$C(18)-C(23)$ & $1.392(8)$ & $\mathrm{C}(46)-\mathrm{C}(47)$ & $1.389(9)$ \\
\hline C(18)-C(19) & $1.392(8)$ & $\mathrm{C}(48)-\mathrm{C}(49)$ & $1.387(6)$ \\
\hline$C(19)-C(20)$ & $1.394(8)$ & $\mathrm{C}(48)-\mathrm{C}(53)$ & $1.405(7)$ \\
\hline$C(19)-C(24)$ & $1.506(8)$ & $\mathrm{C}(49)-\mathrm{C}(50)$ & $1.378(7)$ \\
\hline$C(20)-C(21)$ & $1.370(10)$ & $\mathrm{C}(50)-\mathrm{C}(51)$ & $1.366(8)$ \\
\hline$C(21)-C(22)$ & $1.365(10)$ & $\mathrm{C}(51)-\mathrm{C}(52)$ & $1.373(8)$ \\
\hline
\end{tabular}




\begin{tabular}{|c|c|}
\hline$C(52)-C(53)$ & $1.385(7)$ \\
\hline $\mathrm{C}(54)-\mathrm{C}(59)$ & $1.388(7)$ \\
\hline$C(54)-C(55)$ & $1.394(7)$ \\
\hline$C(55)-C(56)$ & $1.395(8)$ \\
\hline$C(56)-C(57)$ & $1.361(11)$ \\
\hline$C(57)-C(58)$ & $1.337(11)$ \\
\hline $\mathrm{C}(58)-\mathrm{C}(59)$ & $1.420(9)$ \\
\hline$C(60)-C(65)$ & $1.396(7)$ \\
\hline $\mathrm{C}(60)-\mathrm{C}(61)$ & $1.398(6)$ \\
\hline$C(61)-C(62)$ & $1.369(7)$ \\
\hline$C(62)-C(63)$ & $1.369(8)$ \\
\hline$C(63)-C(64)$ & $1.366(8)$ \\
\hline$C(64)-C(65)$ & $1.386(7)$ \\
\hline $\mathrm{O}(1 \mathrm{~S})-\mathrm{C}(1 \mathrm{~S})$ & $1.284(13)$ \\
\hline $\mathrm{O}(1 \mathrm{~S})-\mathrm{C}(3 \mathrm{~S})$ & $1.371(15)$ \\
\hline $\mathrm{C}(1 \mathrm{~S})-\mathrm{C}(2 \mathrm{~S})$ & $1.354(18)$ \\
\hline $\mathrm{C}(3 \mathrm{~S})-\mathrm{C}(4 \mathrm{~S})$ & $1.16(2)$ \\
\hline $\mathrm{C}(10)-\mathrm{Fe}(1)-\mathrm{N}(2)$ & $114.56(18)$ \\
\hline $\mathrm{C}(10)-\mathrm{Fe}(1)-\mathrm{O}(1)$ & $99.86(19)$ \\
\hline $\mathrm{N}(2)-\mathrm{Fe}(1)-\mathrm{O}(1)$ & $145.41(15)$ \\
\hline $\mathrm{C}(10)-\mathrm{Fe}(1)-\mathrm{N}(3)$ & 107.91(17) \\
\hline $\mathrm{N}(2)-\mathrm{Fe}(1)-\mathrm{N}(3)$ & $72.62(13)$ \\
\hline $\mathrm{O}(1)-\mathrm{Fe}(1)-\mathrm{N}(3)$ & $94.33(16)$ \\
\hline $\mathrm{C}(10)-\mathrm{Fe}(1)-\mathrm{N}(1)$ & 107.96(17) \\
\hline $\mathrm{N}(2)-\mathrm{Fe}(1)-\mathrm{N}(1)$ & $72.31(13)$ \\
\hline $\mathrm{O}(1)-\mathrm{Fe}(1)-\mathrm{N}(1)$ & $101.17(17)$ \\
\hline $\mathrm{N}(3)-\mathrm{Fe}(1)-\mathrm{N}(1)$ & $137.54(13)$ \\
\hline $\mathrm{C}(10)-\mathrm{Si}(1)-\mathrm{C}(11)$ & 109.7(3) \\
\hline $\mathrm{C}(10)-\mathrm{Si}(1)-\mathrm{C}(12)$ & $110.4(3)$ \\
\hline $\mathrm{C}(11)-\mathrm{Si}(1)-\mathrm{C}(12)$ & $108.4(5)$ \\
\hline $\mathrm{C}(10)-\mathrm{Si}(1)-\mathrm{C}(13)$ & $115.0(3)$ \\
\hline $\mathrm{C}(11)-\mathrm{Si}(1)-\mathrm{C}(13)$ & $106.4(5)$ \\
\hline $\mathrm{C}(12)-\mathrm{Si}(1)-\mathrm{C}(13)$ & $106.7(4)$ \\
\hline $\mathrm{C}(16)-\mathrm{O}(1)-\mathrm{C}(14)$ & $113.8(6)$ \\
\hline $\mathrm{C}(16)-\mathrm{O}(1)-\mathrm{Fe}(1)$ & $126.1(5)$ \\
\hline
\end{tabular}

\begin{tabular}{|c|c|}
\hline $\mathrm{C}(14)-\mathrm{O}(1)-\mathrm{Fe}(1)$ & $119.3(4)$ \\
\hline $\mathrm{C}(8)-\mathrm{N}(1)-\mathrm{C}(18)$ & $118.4(4)$ \\
\hline $\mathrm{C}(8)-\mathrm{N}(1)-\mathrm{Fe}(1)$ & $112.9(3)$ \\
\hline $\mathrm{C}(18)-\mathrm{N}(1)-\mathrm{Fe}(1)$ & $128.5(3)$ \\
\hline $\mathrm{C}(7)-\mathrm{N}(2)-\mathrm{C}(3)$ & $119.8(3)$ \\
\hline $\mathrm{C}(7)-\mathrm{N}(2)-\mathrm{Fe}(1)$ & $119.8(3)$ \\
\hline $\mathrm{C}(3)-\mathrm{N}(2)-\mathrm{Fe}(1)$ & $118.4(3)$ \\
\hline $\mathrm{C}(2)-\mathrm{N}(3)-\mathrm{C}(30)$ & 119.1(3) \\
\hline $\mathrm{C}(2)-\mathrm{N}(3)-\mathrm{Fe}(1)$ & $113.5(3)$ \\
\hline $\mathrm{C}(30)-\mathrm{N}(3)-\mathrm{Fe}(1)$ & $127.1(3)$ \\
\hline $\mathrm{N}(3)-\mathrm{C}(2)-\mathrm{C}(1)$ & $126.7(4)$ \\
\hline $\mathrm{N}(3)-\mathrm{C}(2)-\mathrm{C}(3)$ & $115.9(4)$ \\
\hline$C(1)-C(2)-C(3)$ & $117.3(4)$ \\
\hline $\mathrm{N}(2)-\mathrm{C}(3)-\mathrm{C}(4)$ & $121.3(4)$ \\
\hline $\mathrm{N}(2)-\mathrm{C}(3)-\mathrm{C}(2)$ & $114.1(3)$ \\
\hline$C(4)-C(3)-C(2)$ & $124.6(4)$ \\
\hline$C(3)-C(4)-C(5)$ & $118.6(4)$ \\
\hline$C(6)-C(5)-C(4)$ & $120.1(4)$ \\
\hline$C(5)-C(6)-C(7)$ & $118.6(4)$ \\
\hline $\mathrm{N}(2)-\mathrm{C}(7)-\mathrm{C}(6)$ & $121.5(4)$ \\
\hline $\mathrm{N}(2)-\mathrm{C}(7)-\mathrm{C}(8)$ & $113.7(4)$ \\
\hline$C(6)-C(7)-C(8)$ & $124.7(4)$ \\
\hline $\mathrm{N}(1)-\mathrm{C}(8)-\mathrm{C}(7)$ & $116.8(4)$ \\
\hline $\mathrm{N}(1)-\mathrm{C}(8)-\mathrm{C}(9)$ & $126.2(4)$ \\
\hline $\mathrm{C}(7)-\mathrm{C}(8)-\mathrm{C}(9)$ & $116.9(4)$ \\
\hline $\mathrm{Si}(1)-\mathrm{C}(10)-\mathrm{Fe}(1)$ & $122.9(3)$ \\
\hline $\mathrm{C}(15)-\mathrm{C}(14)-\mathrm{O}(1)$ & $116.9(8)$ \\
\hline $\mathrm{O}(1)-\mathrm{C}(16)-\mathrm{C}(17)$ & $113.6(9)$ \\
\hline $\mathrm{C}(23)-\mathrm{C}(18)-\mathrm{C}(19)$ & $122.6(5)$ \\
\hline $\mathrm{C}(23)-\mathrm{C}(18)-\mathrm{N}(1)$ & $119.1(5)$ \\
\hline $\mathrm{C}(19)-\mathrm{C}(18)-\mathrm{N}(1)$ & $118.2(5)$ \\
\hline $\mathrm{C}(18)-\mathrm{C}(19)-\mathrm{C}(20)$ & $117.9(6)$ \\
\hline $\mathrm{C}(18)-\mathrm{C}(19)-\mathrm{C}(24)$ & $123.7(5)$ \\
\hline $\mathrm{C}(20)-\mathrm{C}(19)-\mathrm{C}(24)$ & $118.4(6)$ \\
\hline $\mathrm{C}(21)-\mathrm{C}(20)-\mathrm{C}(19)$ & $120.2(7)$ \\
\hline$C(22)-C(21)-C(20)$ & $121.7(6)$ \\
\hline
\end{tabular}




\begin{tabular}{|c|c|}
\hline $\mathrm{C}(21)-\mathrm{C}(22)-\mathrm{C}(23)$ & $120.2(6)$ \\
\hline $\mathrm{C}(18)-\mathrm{C}(23)-\mathrm{C}(22)$ & $117.4(6)$ \\
\hline $\mathrm{C}(18)-\mathrm{C}(23)-\mathrm{C}(27)$ & $122.8(5)$ \\
\hline $\mathrm{C}(22)-\mathrm{C}(23)-\mathrm{C}(27)$ & 119.7(6) \\
\hline$C(26)-C(24)-C(19)$ & $113.3(5)$ \\
\hline $\mathrm{C}(26)-\mathrm{C}(24)-\mathrm{C}(25)$ & $108.4(7)$ \\
\hline$C(19)-C(24)-C(25)$ & $112.9(5)$ \\
\hline $\mathrm{C}(23)-\mathrm{C}(27)-\mathrm{C}(28)$ & $113.8(6)$ \\
\hline$C(23)-C(27)-C(29)$ & $111.2(6)$ \\
\hline $\mathrm{C}(28)-\mathrm{C}(27)-\mathrm{C}(29)$ & $110.0(6)$ \\
\hline$C(31)-C(30)-C(35)$ & $122.9(4)$ \\
\hline $\mathrm{C}(31)-\mathrm{C}(30)-\mathrm{N}(3)$ & $120.2(4)$ \\
\hline $\mathrm{C}(35)-\mathrm{C}(30)-\mathrm{N}(3)$ & $116.8(4)$ \\
\hline$C(30)-C(31)-C(32)$ & $116.5(5)$ \\
\hline$C(30)-C(31)-C(36)$ & 123.1(4) \\
\hline $\mathrm{C}(32)-\mathrm{C}(31)-\mathrm{C}(36)$ & $120.4(5)$ \\
\hline$C(33)-C(32)-C(31)$ & $121.9(5)$ \\
\hline $\mathrm{C}(32)-\mathrm{C}(33)-\mathrm{C}(34)$ & $120.3(5)$ \\
\hline $\mathrm{C}(33)-\mathrm{C}(34)-\mathrm{C}(35)$ & $120.5(5)$ \\
\hline $\mathrm{C}(34)-\mathrm{C}(35)-\mathrm{C}(30)$ & $117.8(5)$ \\
\hline $\mathrm{C}(34)-\mathrm{C}(35)-\mathrm{C}(39)$ & $119.4(5)$ \\
\hline $\mathrm{C}(30)-\mathrm{C}(35)-\mathrm{C}(39)$ & $122.7(4)$ \\
\hline $\mathrm{C}(31)-\mathrm{C}(36)-\mathrm{C}(38)$ & $113.0(4)$ \\
\hline $\mathrm{C}(31)-\mathrm{C}(36)-\mathrm{C}(37)$ & $113.4(5)$ \\
\hline $\mathrm{C}(38)-\mathrm{C}(36)-\mathrm{C}(37)$ & $108.9(5)$ \\
\hline $\mathrm{C}(40)-\mathrm{C}(39)-\mathrm{C}(35)$ & 111.2(5) \\
\hline $\mathrm{C}(40)-\mathrm{C}(39)-\mathrm{C}(41)$ & $108.5(5)$ \\
\hline $\mathrm{C}(35)-\mathrm{C}(39)-\mathrm{C}(41)$ & 113.7(4) \\
\hline $\mathrm{C}(42)-\mathrm{B}(1)-\mathrm{C}(60)$ & 111.2(4) \\
\hline $\mathrm{C}(42)-\mathrm{B}(1)-\mathrm{C}(54)$ & $113.9(4)$ \\
\hline $\mathrm{C}(60)-\mathrm{B}(1)-\mathrm{C}(54)$ & $105.4(4)$ \\
\hline $\mathrm{C}(42)-\mathrm{B}(1)-\mathrm{C}(48)$ & $103.7(4)$ \\
\hline $\mathrm{C}(60)-\mathrm{B}(1)-\mathrm{C}(48)$ & 114.1(4) \\
\hline $\mathrm{C}(54)-\mathrm{B}(1)-\mathrm{C}(48)$ & $108.6(4)$ \\
\hline$C(43)-C(42)-C(47)$ & $114.0(5)$ \\
\hline $\mathrm{C}(43)-\mathrm{C}(42)-\mathrm{B}(1)$ & $120.1(5)$ \\
\hline
\end{tabular}

$\begin{array}{ll}\mathrm{C}(47)-\mathrm{C}(42)-\mathrm{B}(1) & 125.8(5) \\ \mathrm{C}(44)-\mathrm{C}(43)-\mathrm{C}(42) & 124.8(7) \\ \mathrm{C}(45)-\mathrm{C}(44)-\mathrm{C}(43) & 118.7(7) \\ \mathrm{C}(44)-\mathrm{C}(45)-\mathrm{C}(46) & 119.8(6) \\ \mathrm{C}(45)-\mathrm{C}(46)-\mathrm{C}(47) & 120.0(8) \\ \mathrm{C}(42)-\mathrm{C}(47)-\mathrm{C}(46) & 122.7(7) \\ \mathrm{C}(49)-\mathrm{C}(48)-\mathrm{C}(53) & 114.9(4) \\ \mathrm{C}(49)-\mathrm{C}(48)-\mathrm{B}(1) & 126.3(4) \\ \mathrm{C}(53)-\mathrm{C}(48)-\mathrm{B}(1) & 118.8(4) \\ \mathrm{C}(50)-\mathrm{C}(49)-\mathrm{C}(48) & 122.6(5) \\ \mathrm{C}(51)-\mathrm{C}(50)-\mathrm{C}(49) & 120.8(5) \\ \mathrm{C}(50)-\mathrm{C}(51)-\mathrm{C}(52) & 119.3(5) \\ \mathrm{C}(51)-\mathrm{C}(52)-\mathrm{C}(53) & 119.5(5) \\ \mathrm{C}(52)-\mathrm{C}(53)-\mathrm{C}(48) & 122.9(5) \\ \mathrm{C}(59)-\mathrm{C}(54)-\mathrm{C}(55) & 115.0(5) \\ \mathrm{C}(59)-\mathrm{C}(54)-\mathrm{B}(1) & 120.2(5) \\ \mathrm{C}(55)-\mathrm{C}(54)-\mathrm{B}(1) & 124.6(5) \\ \mathrm{C}(56)-\mathrm{C}(55)-\mathrm{C}(54) & 123.8(7) \\ \mathrm{C}(57)-\mathrm{C}(56)-\mathrm{C}(55) & 118.4(8) \\ \mathrm{C}(58)-\mathrm{C}(57)-\mathrm{C}(56) & 121.1(7) \\ \mathrm{C}(57)-\mathrm{C}(58)-\mathrm{C}(59) & 120.3(8) \\ \mathrm{C}(54)-\mathrm{C}(59)-\mathrm{C}(58) & 121.3(7) \\ \mathrm{C}(65)-\mathrm{C}(60)-\mathrm{C}(61) & 113.2(4) \\ \mathrm{C}(65)-\mathrm{C}(60)-\mathrm{B}(1) & 122.6(4) \\ \mathrm{C}(61)-\mathrm{C}(60)-\mathrm{B}(1) & 124.0(4) \\ \mathrm{C}(62)-\mathrm{C}(61)-\mathrm{C}(60) & 123.7(5) \\ \mathrm{C}(61)-\mathrm{C}(62)-\mathrm{C}(63) & 120.9(5) \\ \mathrm{C}(64)-\mathrm{C}(63)-\mathrm{C}(62) & 118.3(5) \\ \mathrm{C}(63)-\mathrm{C}(64)-\mathrm{C}(65) & 120.2(5) \\ \mathrm{C}(64)-\mathrm{C}(65)-\mathrm{C}(60) & 123.7(5) \\ \mathrm{C}(1 \mathrm{~S})-\mathrm{O}(1 \mathrm{~S})-\mathrm{C}(3 \mathrm{~S}) & 122.8(14) \\ \mathrm{O}(1 \mathrm{~S})-\mathrm{C}(1 \mathrm{~S})-\mathrm{C}(2 \mathrm{~S}) & \\ \mathrm{C}(3 \mathrm{~S})-\mathrm{O}(1 \mathrm{~S}) & \end{array}$

Symmetry transformations used to generate equivalent atoms: 
a)

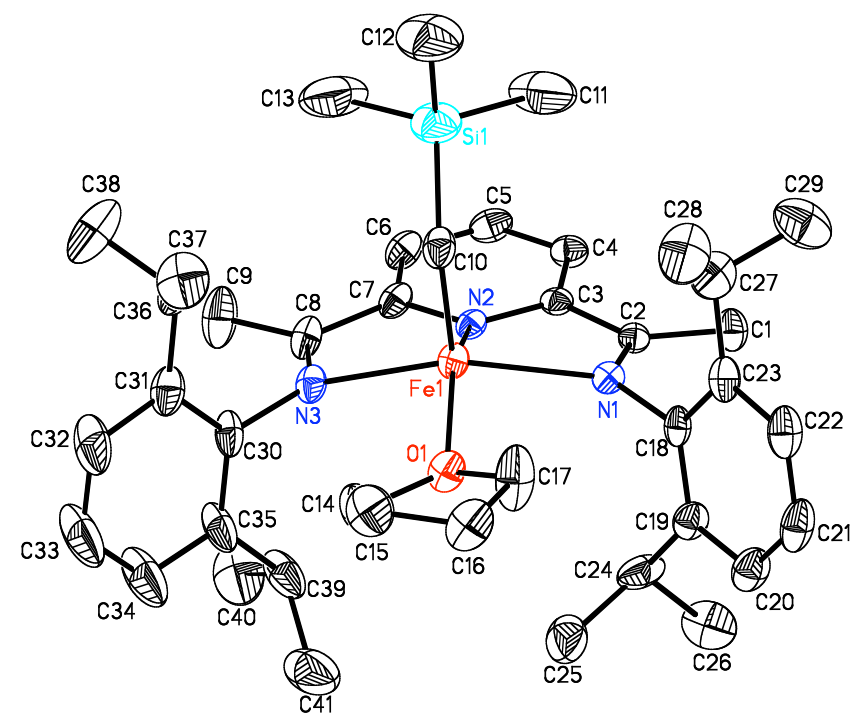

b)

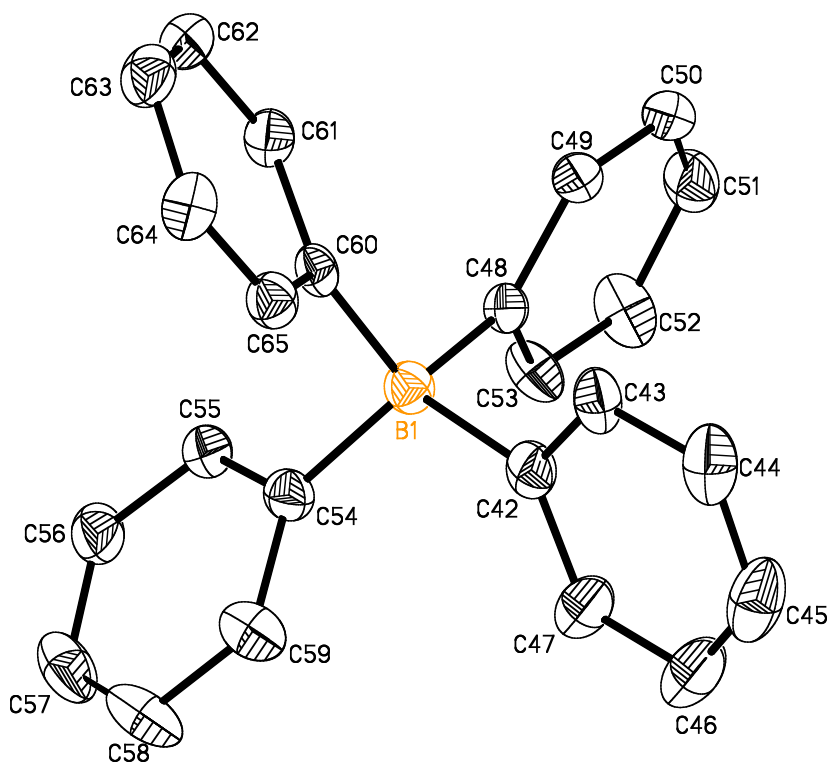

Figure S2. Fully labeled view of a) the cation and b) the anion of $\mathbf{2 - T H F}$ at $30 \%$ probability ellipsoids. Hydrogen atoms omitted for clarity. 
Table S3. Crystal data and structure refinement for 2-THF.

Identification code

Empirical formula

Formula weight

Temperature

Wavelength

Crystal system

Space group

Unit cell dimensions

Volume

Z

Density (calculated)

Absorption coefficient

$\mathrm{F}(000)$

Crystal size

Theta range for data collection

Index ranges

Reflections collected

Independent reflections

Completeness to theta $=22.16^{\circ}$

Absorption correction

Max. and min. transmission

Refinement method

Data / restraints / parameters

Goodness-of-fit on $\mathrm{F}^{2}$

Final $\mathrm{R}$ indices $[\mathrm{I}>2 \operatorname{sigma}(\mathrm{I})]$

$\mathrm{R}$ indices (all data)

Largest diff. peak and hole mwb11

C65 H82 B Fe N3 O Si

1016.09

173(2) K

$0.71073 \AA$

Monoclinic

$\mathrm{P} 2(1) / \mathrm{c}$

$\mathrm{a}=13.0823(4) \AA \quad \alpha=90^{\circ}$.

$\mathrm{b}=23.5818(9) \AA \quad \beta=101.852(1)^{\circ}$.

$\mathrm{c}=21.4652(8) \AA \quad \gamma=90^{\circ}$.

$6480.9(4) \AA^{3}$

4

$1.041 \mathrm{Mg} / \mathrm{m}^{3}$

$0.290 \mathrm{~mm}^{-1}$

2184

$0.70 \times 0.20 \times 0.10 \mathrm{~mm}^{3}$

1.59 to $22.16^{\circ}$.

$-13<=\mathrm{h}<=10,-22<=\mathrm{k}<=24,-22<=\mathrm{l}<=22$

28622

$8093[\mathrm{R}($ int $)=0.0361]$

$99.8 \%$

Semiempirical by SADABS

0.9716 and 0.8228

Full-matrix least-squares on $\mathrm{F}^{2}$

8093 / 0 / 657

1.098

$\mathrm{R} 1=0.0428, \mathrm{wR} 2=0.1164$

$\mathrm{R} 1=0.0595, \mathrm{wR} 2=0.1221$

0.289 and -0.372 e. $\AA^{-3}$ 
Table S4. Bond lengths $[\AA]$ and angles $\left[^{\circ}\right]$ for 2-THF.

\begin{tabular}{|c|c|c|c|}
\hline $\mathrm{Fe}(1)-\mathrm{C}(10)$ & $2.034(3)$ & $C(22)-C(23)$ & $1.398(4)$ \\
\hline $\mathrm{Fe}(1)-\mathrm{N}(2)$ & $2.089(2)$ & $C(23)-C(27)$ & $1.515(4)$ \\
\hline $\mathrm{Fe}(1)-\mathrm{O}(1)$ & $2.1237(18)$ & $C(24)-C(26)$ & $1.528(5)$ \\
\hline $\mathrm{Fe}(1)-\mathrm{N}(1)$ & $2.286(2)$ & $C(24)-C(25)$ & $1.531(5)$ \\
\hline $\mathrm{Fe}(1)-\mathrm{N}(3)$ & $2.328(2)$ & $C(27)-C(29)$ & $1.511(5)$ \\
\hline $\mathrm{Si}(1)-\mathrm{C}(11)$ & $1.856(4)$ & $C(27)-C(28)$ & $1.521(5)$ \\
\hline $\operatorname{Si}(1)-C(12)$ & $1.856(4)$ & $C(30)-C(31)$ & $1.383(4)$ \\
\hline $\operatorname{Si}(1)-C(13)$ & $1.879(4)$ & $C(30)-C(35)$ & $1.400(4)$ \\
\hline $\operatorname{Si}(1)-C(10)$ & $1.887(4)$ & $C(31)-C(32)$ & $1.405(5)$ \\
\hline $\mathrm{O}(1)-\mathrm{C}(14)$ & $1.432(4)$ & $C(31)-C(36)$ & $1.504(4)$ \\
\hline $\mathrm{O}(1)-\mathrm{C}(17)$ & $1.451(3)$ & $C(32)-C(33)$ & $1.358(5)$ \\
\hline $\mathrm{N}(1)-\mathrm{C}(2)$ & $1.284(3)$ & $C(33)-C(34)$ & $1.378(5)$ \\
\hline $\mathrm{N}(1)-\mathrm{C}(18)$ & $1.445(3)$ & $C(34)-C(35)$ & $1.383(5)$ \\
\hline $\mathrm{N}(2)-\mathrm{C}(3)$ & $1.336(3)$ & $C(35)-C(39)$ & $1.521(4)$ \\
\hline $\mathrm{N}(2)-\mathrm{C}(7)$ & $1.345(3)$ & $C(36)-C(37)$ & $1.535(4)$ \\
\hline $\mathrm{N}(3)-\mathrm{C}(8)$ & $1.274(3)$ & $C(36)-C(38)$ & $1.541(4)$ \\
\hline $\mathrm{N}(3)-\mathrm{C}(30)$ & $1.453(3)$ & $C(39)-C(41)$ & $1.506(5)$ \\
\hline$C(1)-C(2)$ & $1.494(3)$ & $C(39)-C(40)$ & $1.546(5)$ \\
\hline$C(2)-C(3)$ & $1.486(3)$ & $C(42)-C(43)$ & $1.395(4)$ \\
\hline $\mathrm{C}(3)-\mathrm{C}(4)$ & $1.388(4)$ & $C(42)-C(47)$ & $1.400(4)$ \\
\hline$C(4)-C(5)$ & $1.381(4)$ & $\mathrm{C}(42)-\mathrm{B}(1)$ & $1.649(4)$ \\
\hline$C(5)-C(6)$ & $1.367(4)$ & $C(43)-C(44)$ & $1.403(4)$ \\
\hline$C(6)-C(7)$ & $1.380(4)$ & $C(44)-C(45)$ & $1.362(5)$ \\
\hline$C(7)-C(8)$ & $1.486(4)$ & $C(45)-C(46)$ & $1.379(5)$ \\
\hline $\mathrm{C}(8)-\mathrm{C}(9)$ & $1.496(4)$ & $C(46)-C(47)$ & $1.395(4)$ \\
\hline$C(14)-C(15)$ & $1.497(5)$ & $C(48)-C(53)$ & $1.391(4)$ \\
\hline$C(15)-C(16)$ & $1.454(4)$ & $C(48)-C(49)$ & $1.398(3)$ \\
\hline$C(16)-C(17)$ & $1.470(4)$ & $\mathrm{C}(48)-\mathrm{B}(1)$ & $1.647(4)$ \\
\hline C(18)-C(19) & $1.388(4)$ & $C(49)-C(50)$ & $1.383(4)$ \\
\hline C(18)-C(23) & $1.400(4)$ & $C(50)-C(51)$ & $1.365(4)$ \\
\hline$C(19)-C(20)$ & $1.389(4)$ & $C(51)-C(52)$ & $1.376(4)$ \\
\hline C(19)-C(24) & $1.524(4)$ & $C(52)-C(53)$ & $1.385(4)$ \\
\hline$C(20)-C(21)$ & $1.370(4)$ & $C(54)-C(55)$ & $1.388(4)$ \\
\hline$C(21)-C(22)$ & $1.362(5)$ & $\mathrm{C}(54)-\mathrm{C}(59)$ & $1.406(4)$ \\
\hline
\end{tabular}




\begin{tabular}{|c|c|c|c|}
\hline $\mathrm{C}(54)-\mathrm{B}(1)$ & $1.635(4)$ & $\mathrm{C}(3)-\mathrm{N}(2)-\mathrm{Fe}(1)$ & $119.14(16)$ \\
\hline $\mathrm{C}(55)-\mathrm{C}(56)$ & $1.386(4)$ & $\mathrm{C}(7)-\mathrm{N}(2)-\mathrm{Fe}(1)$ & $118.44(17)$ \\
\hline $\mathrm{C}(56)-\mathrm{C}(57)$ & $1.384(4)$ & $\mathrm{C}(8)-\mathrm{N}(3)-\mathrm{C}(30)$ & $119.7(2)$ \\
\hline $\mathrm{C}(57)-\mathrm{C}(58)$ & $1.355(5)$ & $\mathrm{C}(8)-\mathrm{N}(3)-\mathrm{Fe}(1)$ & $111.68(18)$ \\
\hline C(58)-C(59) & $1.389(4)$ & $\mathrm{C}(30)-\mathrm{N}(3)-\mathrm{Fe}(1)$ & $128.14(18)$ \\
\hline $\mathrm{C}(60)-\mathrm{C}(65)$ & $1.395(4)$ & $\mathrm{N}(1)-\mathrm{C}(2)-\mathrm{C}(3)$ & $115.3(2)$ \\
\hline $\mathrm{C}(60)-\mathrm{C}(61)$ & $1.396(4)$ & $\mathrm{N}(1)-\mathrm{C}(2)-\mathrm{C}(1)$ & $126.7(2)$ \\
\hline $\mathrm{C}(60)-\mathrm{B}(1)$ & $1.647(4)$ & $\mathrm{C}(3)-\mathrm{C}(2)-\mathrm{C}(1)$ & $117.9(2)$ \\
\hline $\mathrm{C}(61)-\mathrm{C}(62)$ & $1.395(4)$ & $\mathrm{N}(2)-\mathrm{C}(3)-\mathrm{C}(4)$ & $121.4(2)$ \\
\hline $\mathrm{C}(62)-\mathrm{C}(63)$ & $1.370(4)$ & $\mathrm{N}(2)-\mathrm{C}(3)-\mathrm{C}(2)$ & $114.0(2)$ \\
\hline $\mathrm{C}(63)-\mathrm{C}(64)$ & $1.368(4)$ & $\mathrm{C}(4)-\mathrm{C}(3)-\mathrm{C}(2)$ & $124.6(2)$ \\
\hline $\mathrm{C}(64)-\mathrm{C}(65)$ & $1.383(4)$ & $\mathrm{C}(5)-\mathrm{C}(4)-\mathrm{C}(3)$ & $118.3(3)$ \\
\hline & & $\mathrm{C}(6)-\mathrm{C}(5)-\mathrm{C}(4)$ & $120.1(3)$ \\
\hline $\mathrm{C}(10)-\mathrm{Fe}(1)-\mathrm{N}(2)$ & $112.87(13)$ & $\mathrm{C}(5)-\mathrm{C}(6)-\mathrm{C}(7)$ & 119.2(3) \\
\hline $\mathrm{C}(10)-\mathrm{Fe}(1)-\mathrm{O}(1)$ & $100.77(12)$ & $\mathrm{N}(2)-\mathrm{C}(7)-\mathrm{C}(6)$ & $121.0(2)$ \\
\hline $\mathrm{N}(2)-\mathrm{Fe}(1)-\mathrm{O}(1)$ & $146.32(8)$ & $\mathrm{N}(2)-\mathrm{C}(7)-\mathrm{C}(8)$ & $113.8(2)$ \\
\hline $\mathrm{C}(10)-\mathrm{Fe}(1)-\mathrm{N}(1)$ & $108.22(15)$ & $\mathrm{C}(6)-\mathrm{C}(7)-\mathrm{C}(8)$ & $125.2(2)$ \\
\hline $\mathrm{N}(2)-\mathrm{Fe}(1)-\mathrm{N}(1)$ & $72.26(8)$ & $\mathrm{N}(3)-\mathrm{C}(8)-\mathrm{C}(7)$ & $117.2(2)$ \\
\hline $\mathrm{O}(1)-\mathrm{Fe}(1)-\mathrm{N}(1)$ & $96.47(7)$ & $\mathrm{N}(3)-\mathrm{C}(8)-\mathrm{C}(9)$ & $126.4(3)$ \\
\hline $\mathrm{C}(10)-\mathrm{Fe}(1)-\mathrm{N}(3)$ & $107.79(14)$ & $\mathrm{C}(7)-\mathrm{C}(8)-\mathrm{C}(9)$ & $116.4(3)$ \\
\hline $\mathrm{N}(2)-\mathrm{Fe}(1)-\mathrm{N}(3)$ & $72.27(8)$ & $\mathrm{Si}(1)-\mathrm{C}(10)-\mathrm{Fe}(1)$ & $124.4(2)$ \\
\hline $\mathrm{O}(1)-\mathrm{Fe}(1)-\mathrm{N}(3)$ & $99.50(8)$ & $\mathrm{O}(1)-\mathrm{C}(14)-\mathrm{C}(15)$ & 106.1(3) \\
\hline $\mathrm{N}(1)-\mathrm{Fe}(1)-\mathrm{N}(3)$ & $136.78(7)$ & $\mathrm{C}(16)-\mathrm{C}(15)-\mathrm{C}(14)$ & 103.4(3) \\
\hline$C(11)-\operatorname{Si}(1)-C(12)$ & $106.6(2)$ & $\mathrm{C}(15)-\mathrm{C}(16)-\mathrm{C}(17)$ & 104.7(3) \\
\hline$C(11)-\operatorname{Si}(1)-C(13)$ & 106.7(3) & $\mathrm{O}(1)-\mathrm{C}(17)-\mathrm{C}(16)$ & 107.6(3) \\
\hline$C(12)-\operatorname{Si}(1)-C(13)$ & $106.2(2)$ & $C(19)-C(18)-C(23)$ & $122.2(2)$ \\
\hline$C(11)-\operatorname{Si}(1)-C(10)$ & $111.5(2)$ & $\mathrm{C}(19)-\mathrm{C}(18)-\mathrm{N}(1)$ & $117.6(2)$ \\
\hline$C(12)-\operatorname{Si}(1)-C(10)$ & $112.35(19)$ & $\mathrm{C}(23)-\mathrm{C}(18)-\mathrm{N}(1)$ & $120.0(3)$ \\
\hline$C(13)-\operatorname{Si}(1)-C(10)$ & $113.1(2)$ & $\mathrm{C}(20)-\mathrm{C}(19)-\mathrm{C}(18)$ & $117.7(3)$ \\
\hline $\mathrm{C}(14)-\mathrm{O}(1)-\mathrm{C}(17)$ & $107.0(2)$ & $C(20)-C(19)-C(24)$ & $119.9(3)$ \\
\hline C(14)-O(1)-Fe(1) & $123.88(18)$ & $\mathrm{C}(18)-\mathrm{C}(19)-\mathrm{C}(24)$ & $122.5(2)$ \\
\hline $\mathrm{C}(17)-\mathrm{O}(1)-\mathrm{Fe}(1)$ & $120.63(19)$ & $C(21)-C(20)-C(19)$ & $121.3(3)$ \\
\hline $\mathrm{C}(2)-\mathrm{N}(1)-\mathrm{C}(18)$ & $119.0(2)$ & $C(22)-C(21)-C(20)$ & $120.2(3)$ \\
\hline $\mathrm{C}(2)-\mathrm{N}(1)-\mathrm{Fe}(1)$ & $114.62(16)$ & $C(21)-C(22)-C(23)$ & $121.4(3)$ \\
\hline $\mathrm{C}(18)-\mathrm{N}(1)-\mathrm{Fe}(1)$ & $126.39(16)$ & $C(22)-C(23)-C(18)$ & $117.1(3)$ \\
\hline $\mathrm{C}(3)-\mathrm{N}(2)-\mathrm{C}(7)$ & $120.1(2)$ & $\mathrm{C}(22)-\mathrm{C}(23)-\mathrm{C}(27)$ & $120.1(3)$ \\
\hline
\end{tabular}




$\begin{array}{ll}\mathrm{C}(18)-\mathrm{C}(23)-\mathrm{C}(27) & 122.8(3) \\ \mathrm{C}(19)-\mathrm{C}(24)-\mathrm{C}(26) & 112.2(3) \\ \mathrm{C}(19)-\mathrm{C}(24)-\mathrm{C}(25) & 111.1(3) \\ \mathrm{C}(26)-\mathrm{C}(24)-\mathrm{C}(25) & 109.9(3) \\ \mathrm{C}(29)-\mathrm{C}(27)-\mathrm{C}(23) & 110.7(3) \\ \mathrm{C}(29)-\mathrm{C}(27)-\mathrm{C}(28) & 109.6(3) \\ \mathrm{C}(23)-\mathrm{C}(27)-\mathrm{C}(28) & 112.6(3) \\ \mathrm{C}(31)-\mathrm{C}(30)-\mathrm{C}(35) & 122.1(3) \\ \mathrm{C}(31)-\mathrm{C}(30)-\mathrm{N}(3) & 120.5(3) \\ \mathrm{C}(35)-\mathrm{C}(30)-\mathrm{N}(3) & 117.2(2) \\ \mathrm{C}(30)-\mathrm{C}(31)-\mathrm{C}(32) & 117.4(3) \\ \mathrm{C}(30)-\mathrm{C}(31)-\mathrm{C}(36) & 123.0(3) \\ \mathrm{C}(32)-\mathrm{C}(31)-\mathrm{C}(36) & 119.6(3) \\ \mathrm{C}(33)-\mathrm{C}(32)-\mathrm{C}(31) & 121.3(3) \\ \mathrm{C}(32)-\mathrm{C}(33)-\mathrm{C}(34) & 120.4(4) \\ \mathrm{C}(33)-\mathrm{C}(34)-\mathrm{C}(35) & 120.8(4) \\ \mathrm{C}(34)-\mathrm{C}(35)-\mathrm{C}(30) & 118.0(3) \\ \mathrm{C}(34)-\mathrm{C}(35)-\mathrm{C}(39) & 119.2(3) \\ \mathrm{C}(30)-\mathrm{C}(35)-\mathrm{C}(39) & 122.7(3) \\ \mathrm{C}(31)-\mathrm{C}(36)-\mathrm{C}(37) & 112.1(3) \\ \mathrm{C}(31)-\mathrm{C}(36)-\mathrm{C}(38) & 111.2(3) \\ \mathrm{C}(37)-\mathrm{C}(36)-\mathrm{C}(38) & 108.2(3) \\ \mathrm{C}(41)-\mathrm{C}(39)-\mathrm{C}(35) & 112.8(3) \\ \mathrm{C}(41)-\mathrm{C}(39)-\mathrm{C}(40) & 111.2(3) \\ \mathrm{C}(35)-\mathrm{C}(39)-\mathrm{C}(40) & 109.9(3) \\ \mathrm{C}(43)-\mathrm{C}(42)-\mathrm{C}(47) & 115.8(3) \\ \mathrm{C}(43)-\mathrm{C}(42)-\mathrm{B}(1) & 121.6(2) \\ \mathrm{C}(47)-\mathrm{C}(42)-\mathrm{B}(1) & 122.3(3) \\ \mathrm{C}(42)-\mathrm{C}(43)-\mathrm{C}(44) & 122.3(3) \\ \mathrm{C}(45)-\mathrm{C}(44)-\mathrm{C}(43) & 120.0(3) \\ \mathrm{C}(44)-\mathrm{C}(45)-\mathrm{C}(46) & 119.7(3) \\ \mathrm{C}(45)-\mathrm{C}(46)-\mathrm{C}(47) & 120.2(3) \\ & \end{array}$

$\begin{array}{ll}\mathrm{C}(53)-\mathrm{C}(48)-\mathrm{B}(1) & 123.1(2) \\ \mathrm{C}(49)-\mathrm{C}(48)-\mathrm{B}(1) & 122.4(2) \\ \mathrm{C}(50)-\mathrm{C}(49)-\mathrm{C}(48) & 123.0(3) \\ \mathrm{C}(51)-\mathrm{C}(50)-\mathrm{C}(49) & 120.4(3) \\ \mathrm{C}(50)-\mathrm{C}(51)-\mathrm{C}(52) & 119.2(3) \\ \mathrm{C}(51)-\mathrm{C}(52)-\mathrm{C}(53) & 119.6(3) \\ \mathrm{C}(52)-\mathrm{C}(53)-\mathrm{C}(48) & 123.6(3) \\ \mathrm{C}(55)-\mathrm{C}(54)-\mathrm{C}(59) & 114.8(3) \\ \mathrm{C}(55)-\mathrm{C}(54)-\mathrm{B}(1) & 120.8(2) \\ \mathrm{C}(59)-\mathrm{C}(54)-\mathrm{B}(1) & 124.3(3) \\ \mathrm{C}(56)-\mathrm{C}(55)-\mathrm{C}(54) & 123.7(3) \\ \mathrm{C}(57)-\mathrm{C}(56)-\mathrm{C}(55) & 119.0(3) \\ \mathrm{C}(58)-\mathrm{C}(57)-\mathrm{C}(56) & 119.7(3) \\ \mathrm{C}(57)-\mathrm{C}(58)-\mathrm{C}(59) & 120.7(3) \\ \mathrm{C}(58)-\mathrm{C}(59)-\mathrm{C}(54) & 122.1(3) \\ \mathrm{C}(65)-\mathrm{C}(60)-\mathrm{C}(61) & 114.5(2) \\ \mathrm{C}(65)-\mathrm{C}(60)-\mathrm{B}(1) & 121.8(2) \\ \mathrm{C}(61)-\mathrm{C}(60)-\mathrm{B}(1) & 123.6(2) \\ \mathrm{C}(62)-\mathrm{C}(61)-\mathrm{C}(60) & 123.0(3) \\ \mathrm{C}(63)-\mathrm{C}(62)-\mathrm{C}(61) & 119.9(3) \\ \mathrm{C}(64)-\mathrm{C}(63)-\mathrm{C}(62) & 119.0(3) \\ \mathrm{C}(63)-\mathrm{C}(64)-\mathrm{C}(65) & 120.6(3) \\ \mathrm{C}(64)-\mathrm{C}(65)-\mathrm{C}(60) & 123.0(3) \\ \mathrm{C}(54)-\mathrm{B}(1)-\mathrm{C}(60) & 105.3(2) \\ \mathrm{C}(54)-\mathrm{B}(1)-\mathrm{C}(48) & 111.0(2) \\ \mathrm{C}(60)-\mathrm{B}(1)-\mathrm{C}(48) & 111.8(2) \\ \mathrm{C}(54)-\mathrm{B}(1)-\mathrm{C}(42) & 114.4(2) \\ \mathrm{C}(60)-\mathrm{B}(1)-\mathrm{C}(42) & 110.9(2) \\ \mathrm{C}(48)-\mathrm{B}(1)-\mathrm{C}(42) & 103.5(2) \\ & \\ & \end{array}$

Symmetry transformations used to generate equivalent atoms: 
a)

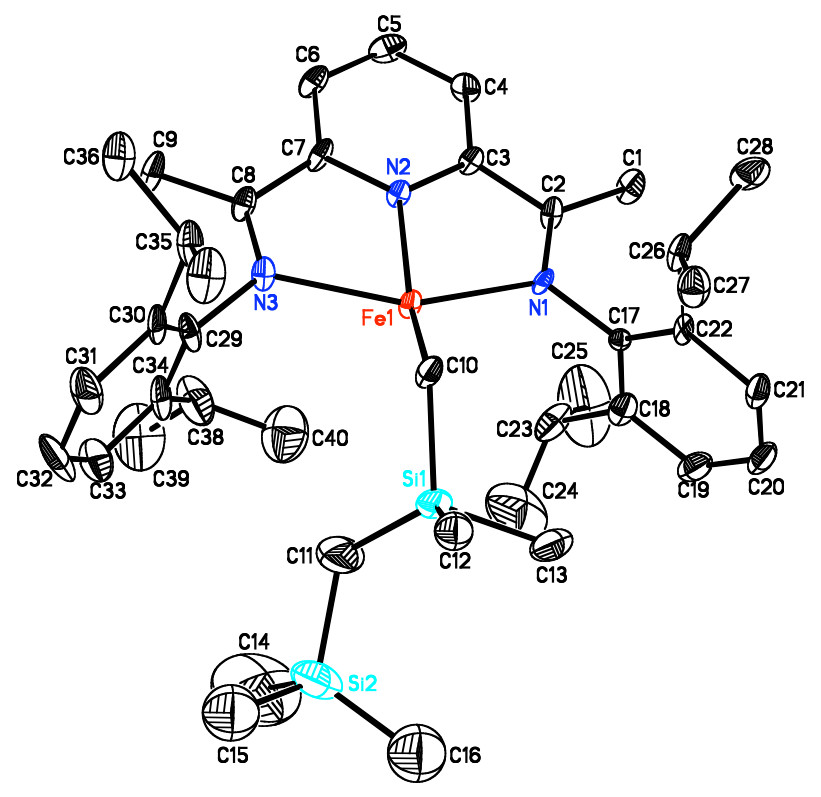

b)

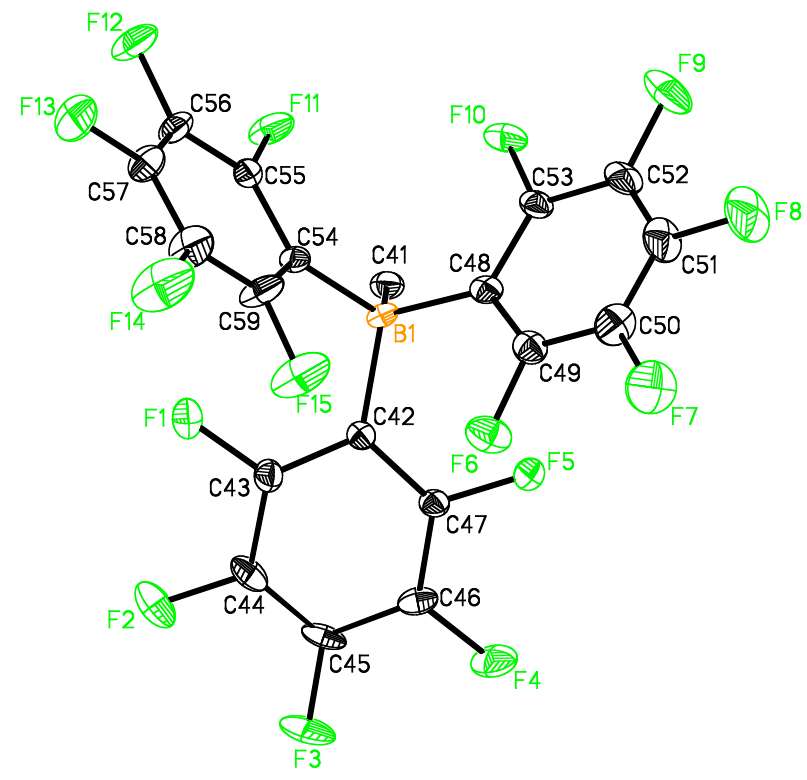

Figure S3. Fully labeled view of the a) cation and b) anion of $\mathbf{3}$ at $30 \%$ probability ellipsoids. Hydrogen atoms omitted for clarity. 
Table S5. Crystal data and structure refinement for 3 .

Identification code

Empirical formula

Formula weight

Temperature

Wavelength

Crystal system

Space group

Unit cell dimensions

Volume

Z

Density (calculated)

Absorption coefficient

$\mathrm{F}(000)$

Crystal size

Theta range for data collection

Index ranges

Reflections collected

Independent reflections

Completeness to theta $=20.83^{\circ}$

Absorption correction

Max. and min. transmission

Refinement method

Data / restraints / parameters

Goodness-of-fit on $\mathrm{F}^{2}$

Final R indices [I $>2 \operatorname{sigma}(\mathrm{I})]$

$\mathrm{R}$ indices (all data)

Largest diff. peak and hole mwb12

C59 H65 B F15 Fe N3 Si2

1223.98

173(2) K

$0.71073 \AA$

Monoclinic

$\mathrm{P} 2(1) / \mathrm{c}$

$\mathrm{a}=15.1477(13) \AA$

$\alpha=90^{\circ}$.

$\mathrm{b}=18.5854(13) \AA$

$\beta=96.923(2)^{\circ}$.

$\mathrm{c}=22.0858(18) \AA$

$\gamma=90^{\circ}$.

6172.4(8) $\AA^{3}$

4

$1.317 \mathrm{Mg} / \mathrm{m}^{3}$

$0.367 \mathrm{~mm}^{-1}$

2536

$0.30 \times 0.10 \times 0.03 \mathrm{~mm}^{3}$

1.74 to $20.83^{\circ}$.

$-15<=\mathrm{h}<=15,-18<=\mathrm{k}<=16,-21<=1<=22$

29811

$6446[\mathrm{R}(\mathrm{int})=0.0815]$

$99.6 \%$

Semiempirical by SADABS

0.9891 and 0.8978

Full-matrix least-squares on $\mathrm{F}^{2}$

6446 / 666 / 733

1.030

$\mathrm{R} 1=0.0633, \mathrm{wR} 2=0.1609$

$\mathrm{R} 1=0.1020, \mathrm{wR} 2=0.1765$

1.112 and -0.623 e. $\AA^{-3}$ 
Table S6. Bond lengths $[\AA]$ and angles $\left[^{\circ}\right]$ for 3 .

\begin{tabular}{|c|c|c|c|}
\hline $\mathrm{Fe}(1)-\mathrm{C}(10)$ & $2.011(6)$ & $C(20)-C(21)$ & $1.377(8)$ \\
\hline $\mathrm{Fe}(1)-\mathrm{N}(2)$ & $2.121(4)$ & $C(21)-C(22)$ & $1.396(8)$ \\
\hline $\mathrm{Fe}(1)-\mathrm{N}(1)$ & $2.217(4)$ & $C(22)-C(26)$ & $1.515(8)$ \\
\hline $\mathrm{Fe}(1)-\mathrm{N}(3)$ & $2.221(4)$ & $C(23)-C(25)$ & $1.448(11)$ \\
\hline $\mathrm{Si}(1)-\mathrm{C}\left(12^{\prime}\right)$ & $1.815(10)$ & $C(23)-C(24)$ & $1.514(11)$ \\
\hline $\operatorname{Si}(1)-C(13)$ & $1.851(7)$ & $C(26)-C(27)$ & $1.516(8)$ \\
\hline $\operatorname{Si}(1)-C(10)$ & $1.855(6)$ & $C(26)-C(28)$ & $1.536(8)$ \\
\hline $\operatorname{Si}(1)-C(11)$ & $1.841(8)$ & $\mathrm{C}(29)-\mathrm{C}(30)$ & $1.382(8)$ \\
\hline $\operatorname{Si}(1)-C(12)$ & $1.972(17)$ & $C(29)-C(34)$ & $1.405(8)$ \\
\hline $\operatorname{Si}(2)-C\left(16^{\prime}\right)$ & $1.66(3)$ & $\mathrm{C}(30)-\mathrm{C}(31)$ & $1.405(8)$ \\
\hline $\operatorname{Si}(2)-C(15)$ & $1.762(16)$ & $\mathrm{C}(30)-\mathrm{C}(35)$ & $1.526(8)$ \\
\hline $\operatorname{Si}(2)-C(16)$ & $1.779(13)$ & $\mathrm{C}(31)-\mathrm{C}(32)$ & $1.387(9)$ \\
\hline $\mathrm{Si}(2)-\mathrm{C}\left(15^{\prime}\right)$ & $1.79(2)$ & $\mathrm{C}(32)-\mathrm{C}(33)$ & $1.369(10)$ \\
\hline $\operatorname{Si}(2)-C(11)$ & $1.842(8)$ & $\mathrm{C}(33)-\mathrm{C}(34)$ & $1.398(9)$ \\
\hline $\operatorname{Si}(2)-C(14)$ & $2.022(14)$ & $\mathrm{C}(34)-\mathrm{C}(38)$ & $1.529(10)$ \\
\hline $\mathrm{N}(1)-\mathrm{C}(2)$ & $1.281(7)$ & $C(35)-C(37)$ & $1.502(8)$ \\
\hline $\mathrm{N}(1)-\mathrm{C}(17)$ & $1.450(7)$ & $C(35)-C(36)$ & $1.538(9)$ \\
\hline $\mathrm{N}(2)-\mathrm{C}(3)$ & $1.339(7)$ & $C(38)-C(39)$ & $1.455(9)$ \\
\hline $\mathrm{N}(2)-\mathrm{C}(7)$ & $1.342(7)$ & $\mathrm{C}(38)-\mathrm{C}(40)$ & $1.488(10)$ \\
\hline $\mathrm{N}(3)-\mathrm{C}(8)$ & $1.280(7)$ & $\mathrm{F}(1)-\mathrm{C}(43)$ & $1.358(7)$ \\
\hline $\mathrm{N}(3)-\mathrm{C}(29)$ & $1.456(7)$ & $\mathrm{F}(2)-\mathrm{C}(44)$ & $1.352(7)$ \\
\hline$C(1)-C(2)$ & $1.482(7)$ & $\mathrm{F}(3)-\mathrm{C}(45)$ & $1.332(7)$ \\
\hline$C(2)-C(3)$ & $1.490(8)$ & $\mathrm{F}(4)-\mathrm{C}(46)$ & $1.351(7)$ \\
\hline$C(3)-C(4)$ & $1.389(8)$ & $\mathrm{F}(5)-\mathrm{C}(47)$ & $1.352(7)$ \\
\hline$C(4)-C(5)$ & $1.378(8)$ & $\mathrm{F}(6)-\mathrm{C}(49)$ & $1.356(7)$ \\
\hline$C(5)-C(6)$ & $1.382(8)$ & $\mathrm{F}(7)-\mathrm{C}(50)$ & $1.361(8)$ \\
\hline$C(6)-C(7)$ & $1.379(8)$ & $\mathrm{F}(8)-\mathrm{C}(51)$ & $1.364(8)$ \\
\hline$C(7)-C(8)$ & $1.478(8)$ & $\mathrm{F}(9)-\mathrm{C}(52)$ & $1.348(8)$ \\
\hline $\mathrm{C}(8)-\mathrm{C}(9)$ & $1.496(8)$ & $\mathrm{F}(10)-\mathrm{C}(53)$ & $1.340(7)$ \\
\hline$C(17)-C(22)$ & $1.375(7)$ & $\mathrm{F}(11)-\mathrm{C}(55)$ & $1.350(7)$ \\
\hline$C(17)-C(18)$ & $1.407(8)$ & $\mathrm{F}(12)-\mathrm{C}(56)$ & $1.362(7)$ \\
\hline C(18)-C(19) & $1.409(8)$ & $\mathrm{F}(13)-\mathrm{C}(57)$ & $1.355(7)$ \\
\hline$C(18)-C(23)$ & $1.525(9)$ & $\mathrm{F}(14)-\mathrm{C}(58)$ & $1.360(8)$ \\
\hline$C(19)-C(20)$ & $1.374(8)$ & $\mathrm{F}(15)-\mathrm{C}(59)$ & $1.360(7)$ \\
\hline
\end{tabular}




\begin{tabular}{|c|c|c|c|}
\hline $\mathrm{C}(41)-\mathrm{B}(1)$ & $1.623(9)$ & $\mathrm{C}(13)-\mathrm{Si}(1)-\mathrm{C}(12)$ & $97.6(7)$ \\
\hline $\mathrm{C}(42)-\mathrm{C}(43)$ & $1.379(8)$ & $\mathrm{C}(10)-\mathrm{Si}(1)-\mathrm{C}(12)$ & $104.1(6)$ \\
\hline$C(42)-C(47)$ & $1.389(8)$ & $\mathrm{C}(11)-\mathrm{Si}(1)-\mathrm{C}(12)$ & $124.2(9)$ \\
\hline $\mathrm{C}(42)-\mathrm{B}(1)$ & $1.661(9)$ & $\mathrm{C}\left(16^{\prime}\right)-\mathrm{Si}(2)-\mathrm{C}(15)$ & $147.9(13)$ \\
\hline $\mathrm{C}(43)-\mathrm{C}(44)$ & $1.391(9)$ & $\mathrm{C}\left(16^{\prime}\right)-\mathrm{Si}(2)-\mathrm{C}(16)$ & $45.9(11)$ \\
\hline $\mathrm{C}(44)-\mathrm{C}(45)$ & $1.346(9)$ & $\mathrm{C}(15)-\mathrm{Si}(2)-\mathrm{C}(16)$ & $114.2(8)$ \\
\hline$C(45)-C(46)$ & $1.367(9)$ & $\mathrm{C}\left(16^{\prime}\right)-\mathrm{Si}(2)-\mathrm{C}\left(15^{\prime}\right)$ & $129.7(16)$ \\
\hline$C(46)-C(47)$ & $1.376(9)$ & $\mathrm{C}(15)-\mathrm{Si}(2)-\mathrm{C}\left(15^{\prime}\right)$ & 29.9(9) \\
\hline $\mathrm{C}(48)-\mathrm{C}(53)$ & $1.364(9)$ & $\mathrm{C}(16)-\mathrm{Si}(2)-\mathrm{C}\left(15^{\prime}\right)$ & $122.2(10)$ \\
\hline C(48)-C(49) & $1.397(8)$ & $C\left(16^{\prime}\right)-\operatorname{Si}(2)-C(11)$ & $106.8(11)$ \\
\hline $\mathrm{C}(48)-\mathrm{B}(1)$ & $1.673(9)$ & $\mathrm{C}(15)-\mathrm{Si}(2)-\mathrm{C}(11)$ & $105.0(8)$ \\
\hline $\mathrm{C}(49)-\mathrm{C}(50)$ & $1.363(9)$ & $\mathrm{C}(16)-\mathrm{Si}(2)-\mathrm{C}(11)$ & $115.8(5)$ \\
\hline$C(50)-C(51)$ & $1.345(10)$ & $\mathrm{C}\left(15^{\prime}\right)-\mathrm{Si}(2)-\mathrm{C}(11)$ & $117.3(10)$ \\
\hline$C(51)-C(52)$ & $1.382(10)$ & $\mathrm{C}\left(16^{\prime}\right)-\mathrm{Si}(2)-\mathrm{C}(14)$ & $67.2(12)$ \\
\hline$C(52)-C(53)$ & $1.389(9)$ & $\mathrm{C}(15)-\mathrm{Si}(2)-\mathrm{C}(14)$ & 107.1(11) \\
\hline$C(54)-C(55)$ & $1.385(8)$ & $\mathrm{C}(16)-\mathrm{Si}(2)-\mathrm{C}(14)$ & $107.0(7)$ \\
\hline$C(54)-C(59)$ & $1.385(9)$ & $\mathrm{C}\left(15^{\prime}\right)-\mathrm{Si}(2)-\mathrm{C}(14)$ & $77.3(15)$ \\
\hline $\mathrm{C}(54)-\mathrm{B}(1)$ & $1.645(9)$ & $\mathrm{C}(11)-\mathrm{Si}(2)-\mathrm{C}(14)$ & $107.3(6)$ \\
\hline$C(55)-C(56)$ & $1.359(9)$ & $\mathrm{C}(2)-\mathrm{N}(1)-\mathrm{C}(17)$ & $117.7(4)$ \\
\hline$C(56)-C(57)$ & $1.348(9)$ & $\mathrm{C}(2)-\mathrm{N}(1)-\mathrm{Fe}(1)$ & $118.4(4)$ \\
\hline $\mathrm{C}(57)-\mathrm{C}(58)$ & $1.368(9)$ & $\mathrm{C}(17)-\mathrm{N}(1)-\mathrm{Fe}(1)$ & $123.8(3)$ \\
\hline \multirow[t]{2}{*}{$\mathrm{C}(58)-\mathrm{C}(59)$} & $1.360(9)$ & $\mathrm{C}(3)-\mathrm{N}(2)-\mathrm{C}(7)$ & $120.2(5)$ \\
\hline & & $\mathrm{C}(3)-\mathrm{N}(2)-\mathrm{Fe}(1)$ & $119.3(4)$ \\
\hline $\mathrm{C}(10)-\mathrm{Fe}(1)-\mathrm{N}(2)$ & $156.1(2)$ & $\mathrm{C}(7)-\mathrm{N}(2)-\mathrm{Fe}(1)$ & $120.2(4)$ \\
\hline $\mathrm{C}(10)-\mathrm{Fe}(1)-\mathrm{N}(1)$ & $108.3(2)$ & $\mathrm{C}(8)-\mathrm{N}(3)-\mathrm{C}(29)$ & $119.8(5)$ \\
\hline $\mathrm{N}(2)-\mathrm{Fe}(1)-\mathrm{N}(1)$ & $72.68(17)$ & $\mathrm{C}(8)-\mathrm{N}(3)-\mathrm{Fe}(1)$ & $118.0(4)$ \\
\hline $\mathrm{C}(10)-\mathrm{Fe}(1)-\mathrm{N}(3)$ & $108.5(2)$ & $\mathrm{C}(29)-\mathrm{N}(3)-\mathrm{Fe}(1)$ & $122.2(4)$ \\
\hline $\mathrm{N}(2)-\mathrm{Fe}(1)-\mathrm{N}(3)$ & $72.22(17)$ & $\mathrm{N}(1)-\mathrm{C}(2)-\mathrm{C}(1)$ & $127.2(5)$ \\
\hline $\mathrm{N}(1)-\mathrm{Fe}(1)-\mathrm{N}(3)$ & $143.01(17)$ & $\mathrm{N}(1)-\mathrm{C}(2)-\mathrm{C}(3)$ & $114.3(5)$ \\
\hline$C\left(12^{\prime}\right)-\operatorname{Si}(1)-C(13)$ & $113.2(5)$ & $C(1)-C(2)-C(3)$ & $118.5(5)$ \\
\hline $\mathrm{C}\left(12^{\prime}\right)-\mathrm{Si}(1)-\mathrm{C}(10)$ & $113.4(4)$ & $\mathrm{N}(2)-\mathrm{C}(3)-\mathrm{C}(4)$ & $120.5(5)$ \\
\hline C(13)-Si(1)-C(10) & $109.5(3)$ & $\mathrm{N}(2)-\mathrm{C}(3)-\mathrm{C}(2)$ & $114.3(5)$ \\
\hline $\mathrm{C}\left(12^{\prime}\right)-\mathrm{Si}(1)-\mathrm{C}(11)$ & $100.0(8)$ & $C(4)-C(3)-C(2)$ & $125.1(5)$ \\
\hline $\mathrm{C}(13)-\mathrm{Si}(1)-\mathrm{C}(11)$ & $110.5(4)$ & $C(5)-C(4)-C(3)$ & $119.5(5)$ \\
\hline$C(10)-\operatorname{Si}(1)-C(11)$ & $109.9(3)$ & $C(4)-C(5)-C(6)$ & $119.5(5)$ \\
\hline $\mathrm{C}\left(12^{\prime}\right)-\mathrm{Si}(1)-\mathrm{C}(12)$ & $24.5(5)$ & $C(7)-C(6)-C(5)$ & $118.6(5)$ \\
\hline
\end{tabular}




\begin{tabular}{|c|c|}
\hline $\mathrm{N}(2)-\mathrm{C}(7)-\mathrm{C}(6)$ & $121.8(5)$ \\
\hline $\mathrm{N}(2)-\mathrm{C}(7)-\mathrm{C}(8)$ & $113.1(5)$ \\
\hline$C(6)-C(7)-C(8)$ & $125.1(5)$ \\
\hline $\mathrm{N}(3)-\mathrm{C}(8)-\mathrm{C}(7)$ & $115.6(5)$ \\
\hline $\mathrm{N}(3)-\mathrm{C}(8)-\mathrm{C}(9)$ & $126.0(5)$ \\
\hline $\mathrm{C}(7)-\mathrm{C}(8)-\mathrm{C}(9)$ & $118.3(5)$ \\
\hline $\operatorname{Si}(1)-C(10)-F e(1)$ & 126.1(3) \\
\hline $\operatorname{Si}(2)-C(11)-\operatorname{Si}(1)$ & $125.1(5)$ \\
\hline$C(22)-C(17)-C(18)$ & $122.5(5)$ \\
\hline $\mathrm{C}(22)-\mathrm{C}(17)-\mathrm{N}(1)$ & $121.0(5)$ \\
\hline $\mathrm{C}(18)-\mathrm{C}(17)-\mathrm{N}(1)$ & $116.2(5)$ \\
\hline $\mathrm{C}(17)-\mathrm{C}(18)-\mathrm{C}(19)$ & $117.3(5)$ \\
\hline $\mathrm{C}(17)-\mathrm{C}(18)-\mathrm{C}(23)$ & $122.8(5)$ \\
\hline $\mathrm{C}(19)-\mathrm{C}(18)-\mathrm{C}(23)$ & $119.9(6)$ \\
\hline$C(20)-C(19)-C(18)$ & $120.5(6)$ \\
\hline $\mathrm{C}(19)-\mathrm{C}(20)-\mathrm{C}(21)$ & $120.3(6)$ \\
\hline$C(20)-C(21)-C(22)$ & $121.3(5)$ \\
\hline $\mathrm{C}(17)-\mathrm{C}(22)-\mathrm{C}(21)$ & $117.8(5)$ \\
\hline $\mathrm{C}(17)-\mathrm{C}(22)-\mathrm{C}(26)$ & $123.0(5)$ \\
\hline $\mathrm{C}(21)-\mathrm{C}(22)-\mathrm{C}(26)$ & $119.2(5)$ \\
\hline $\mathrm{C}(25)-\mathrm{C}(23)-\mathrm{C}(24)$ & $109.9(8)$ \\
\hline $\mathrm{C}(25)-\mathrm{C}(23)-\mathrm{C}(18)$ & $112.4(6)$ \\
\hline $\mathrm{C}(24)-\mathrm{C}(23)-\mathrm{C}(18)$ & 111.2(6) \\
\hline $\mathrm{C}(22)-\mathrm{C}(26)-\mathrm{C}(27)$ & $113.2(5)$ \\
\hline $\mathrm{C}(22)-\mathrm{C}(26)-\mathrm{C}(28)$ & $111.7(5)$ \\
\hline $\mathrm{C}(27)-\mathrm{C}(26)-\mathrm{C}(28)$ & $109.8(5)$ \\
\hline $\mathrm{C}(30)-\mathrm{C}(29)-\mathrm{C}(34)$ & $124.3(5)$ \\
\hline $\mathrm{C}(30)-\mathrm{C}(29)-\mathrm{N}(3)$ & $119.4(5)$ \\
\hline C(34)-C(29)-N(3) & $116.1(5)$ \\
\hline $\mathrm{C}(29)-\mathrm{C}(30)-\mathrm{C}(31)$ & $117.6(6)$ \\
\hline$C(29)-C(30)-C(35)$ & $122.6(5)$ \\
\hline $\mathrm{C}(31)-\mathrm{C}(30)-\mathrm{C}(35)$ & $119.7(6)$ \\
\hline $\mathrm{C}(32)-\mathrm{C}(31)-\mathrm{C}(30)$ & $119.4(6)$ \\
\hline $\mathrm{C}(33)-\mathrm{C}(32)-\mathrm{C}(31)$ & $121.3(6)$ \\
\hline $\mathrm{C}(32)-\mathrm{C}(33)-\mathrm{C}(34)$ & $121.7(7)$ \\
\hline$C(33)-C(34)-C(29)$ & $115.5(6)$ \\
\hline
\end{tabular}

\begin{tabular}{|c|c|}
\hline $\mathrm{C}(33)-\mathrm{C}(34)-\mathrm{C}(38)$ & $121.5(6)$ \\
\hline $\mathrm{C}(29)-\mathrm{C}(34)-\mathrm{C}(38)$ & $123.0(6)$ \\
\hline $\mathrm{C}(37)-\mathrm{C}(35)-\mathrm{C}(30)$ & $113.6(5)$ \\
\hline $\mathrm{C}(37)-\mathrm{C}(35)-\mathrm{C}(36)$ & $109.3(5)$ \\
\hline $\mathrm{C}(30)-\mathrm{C}(35)-\mathrm{C}(36)$ & $111.7(5)$ \\
\hline $\mathrm{C}(39)-\mathrm{C}(38)-\mathrm{C}(40)$ & 111.1(7) \\
\hline $\mathrm{C}(39)-\mathrm{C}(38)-\mathrm{C}(34)$ & $117.0(6)$ \\
\hline $\mathrm{C}(40)-\mathrm{C}(38)-\mathrm{C}(34)$ & $114.4(7)$ \\
\hline $\mathrm{C}(43)-\mathrm{C}(42)-\mathrm{C}(47)$ & $112.1(6)$ \\
\hline $\mathrm{C}(43)-\mathrm{C}(42)-\mathrm{B}(1)$ & $123.6(5)$ \\
\hline $\mathrm{C}(47)-\mathrm{C}(42)-\mathrm{B}(1)$ & $122.9(5)$ \\
\hline F(1)-C(43)-C(42) & $120.5(6)$ \\
\hline $\mathrm{F}(1)-\mathrm{C}(43)-\mathrm{C}(44)$ & $114.9(6)$ \\
\hline $\mathrm{C}(42)-\mathrm{C}(43)-\mathrm{C}(44)$ & $124.6(6)$ \\
\hline $\mathrm{C}(45)-\mathrm{C}(44)-\mathrm{F}(2)$ & $120.1(6)$ \\
\hline $\mathrm{C}(45)-\mathrm{C}(44)-\mathrm{C}(43)$ & $120.1(6)$ \\
\hline $\mathrm{F}(2)-\mathrm{C}(44)-\mathrm{C}(43)$ & $119.7(6)$ \\
\hline $\mathrm{F}(3)-\mathrm{C}(45)-\mathrm{C}(44)$ & $121.4(7)$ \\
\hline $\mathrm{F}(3)-\mathrm{C}(45)-\mathrm{C}(46)$ & $120.2(6)$ \\
\hline $\mathrm{C}(44)-\mathrm{C}(45)-\mathrm{C}(46)$ & $118.4(6)$ \\
\hline $\mathrm{F}(4)-\mathrm{C}(46)-\mathrm{C}(45)$ & $119.8(6)$ \\
\hline $\mathrm{F}(4)-\mathrm{C}(46)-\mathrm{C}(47)$ & $120.0(6)$ \\
\hline$C(45)-C(46)-C(47)$ & $120.0(6)$ \\
\hline $\mathrm{F}(5)-\mathrm{C}(47)-\mathrm{C}(46)$ & $115.6(5)$ \\
\hline $\mathrm{F}(5)-\mathrm{C}(47)-\mathrm{C}(42)$ & $119.9(5)$ \\
\hline$C(46)-C(47)-C(42)$ & $124.6(6)$ \\
\hline $\mathrm{C}(53)-\mathrm{C}(48)-\mathrm{C}(49)$ & $112.7(6)$ \\
\hline $\mathrm{C}(53)-\mathrm{C}(48)-\mathrm{B}(1)$ & $121.1(5)$ \\
\hline $\mathrm{C}(49)-\mathrm{C}(48)-\mathrm{B}(1)$ & $126.2(6)$ \\
\hline $\mathrm{F}(6)-\mathrm{C}(49)-\mathrm{C}(50)$ & $115.4(6)$ \\
\hline $\mathrm{F}(6)-\mathrm{C}(49)-\mathrm{C}(48)$ & $119.6(6)$ \\
\hline $\mathrm{C}(50)-\mathrm{C}(49)-\mathrm{C}(48)$ & $124.9(7)$ \\
\hline $\mathrm{C}(51)-\mathrm{C}(50)-\mathrm{F}(7)$ & $120.4(7)$ \\
\hline $\mathrm{C}(51)-\mathrm{C}(50)-\mathrm{C}(49)$ & $120.0(7)$ \\
\hline $\mathrm{F}(7)-\mathrm{C}(50)-\mathrm{C}(49)$ & $119.6(7)$ \\
\hline $\mathrm{C}(50)-\mathrm{C}(51)-\mathrm{F}(8)$ & $121.6(7)$ \\
\hline
\end{tabular}




\begin{tabular}{|c|c|c|c|}
\hline $\mathrm{C}(50)-\mathrm{C}(51)-\mathrm{C}(52)$ & $118.8(7)$ & $\mathrm{F}(13)-\mathrm{C}(57)-\mathrm{C}(58)$ & $120.6(7)$ \\
\hline $\mathrm{F}(8)-\mathrm{C}(51)-\mathrm{C}(52)$ & $119.6(7)$ & $\mathrm{F}(14)-\mathrm{C}(58)-\mathrm{C}(59)$ & $121.5(6)$ \\
\hline $\mathrm{F}(9)-\mathrm{C}(52)-\mathrm{C}(51)$ & $120.1(6)$ & $\mathrm{F}(14)-\mathrm{C}(58)-\mathrm{C}(57)$ & $119.2(7)$ \\
\hline F(9)-C(52)-C(53) & $120.7(7)$ & $\mathrm{C}(59)-\mathrm{C}(58)-\mathrm{C}(57)$ & $119.2(7)$ \\
\hline $\mathrm{C}(51)-\mathrm{C}(52)-\mathrm{C}(53)$ & $119.2(7)$ & $\mathrm{C}(58)-\mathrm{C}(59)-\mathrm{F}(15)$ & $115.4(6)$ \\
\hline $\mathrm{F}(10)-\mathrm{C}(53)-\mathrm{C}(48)$ & $122.1(6)$ & $\mathrm{C}(58)-\mathrm{C}(59)-\mathrm{C}(54)$ & $126.2(6)$ \\
\hline $\mathrm{F}(10)-\mathrm{C}(53)-\mathrm{C}(52)$ & $113.5(6)$ & $F(15)-C(59)-C(54)$ & $118.4(6)$ \\
\hline $\mathrm{C}(48)-\mathrm{C}(53)-\mathrm{C}(52)$ & $124.4(6)$ & $\mathrm{C}(41)-\mathrm{B}(1)-\mathrm{C}(54)$ & $114.1(5)$ \\
\hline $\mathrm{C}(55)-\mathrm{C}(54)-\mathrm{C}(59)$ & $110.6(6)$ & $\mathrm{C}(41)-\mathrm{B}(1)-\mathrm{C}(42)$ & $100.3(5)$ \\
\hline $\mathrm{C}(55)-\mathrm{C}(54)-\mathrm{B}(1)$ & $125.9(6)$ & $\mathrm{C}(54)-\mathrm{B}(1)-\mathrm{C}(42)$ & $111.6(5)$ \\
\hline $\mathrm{C}(59)-\mathrm{C}(54)-\mathrm{B}(1)$ & $123.4(5)$ & $\mathrm{C}(41)-\mathrm{B}(1)-\mathrm{C}(48)$ & $112.8(5)$ \\
\hline $\mathrm{F}(11)-\mathrm{C}(55)-\mathrm{C}(56)$ & $114.7(5)$ & C(54)-B(1)-C(48) & $105.3(5)$ \\
\hline $\mathrm{F}(11)-\mathrm{C}(55)-\mathrm{C}(54)$ & $120.0(6)$ & $\mathrm{C}(42)-\mathrm{B}(1)-\mathrm{C}(48)$ & $113.0(5)$ \\
\hline $\mathrm{C}(56)-\mathrm{C}(55)-\mathrm{C}(54)$ & $125.3(6)$ & & \\
\hline $\mathrm{C}(57)-\mathrm{C}(56)-\mathrm{C}(55)$ & $120.6(6)$ & & \\
\hline $\mathrm{C}(57)-\mathrm{C}(56)-\mathrm{F}(12)$ & $118.5(6)$ & \multirow{4}{*}{\multicolumn{2}{|c|}{$\begin{array}{l}\text { Symmetry transforn } \\
\text { equivalent atoms: }\end{array}$}} \\
\hline $\mathrm{C}(55)-\mathrm{C}(56)-\mathrm{F}(12)$ & $120.8(6)$ & & \\
\hline $\mathrm{C}(56)-\mathrm{C}(57)-\mathrm{F}(13)$ & $121.4(6)$ & & \\
\hline $\mathrm{C}(56)-\mathrm{C}(57)-\mathrm{C}(58)$ & $118.0(6)$ & & \\
\hline
\end{tabular}

\section{References.}

${ }^{1}$ Pangborn, A.B.; Giardello, M.A.; Grubbs, R.H.; Rosen, R.K.; Timmers, F.J. Organometallics 1996, 15, 1518.

${ }^{2}$ Eshuis, J. J. W.; Tan, Y. Y.; Meetsma, A.; Teuben, J. H.; Renkema, H.; Evens, G. G. Organometallics 1992, 11, 362.

${ }^{3}$ Bouwkamp, M. W.; Bart, S. C.; Hawrelak, E. J.; Trovitch, R. J.; Lobkovsky, E.; Chirik, P. J. Chem. Commun. submitted. 